KRzyszTof TUREK (Kraków)

\title{
OPTION PRICING IN A CEV MODEL WITH LIQUIDITY COSTS
}

Abstract. The goal of this paper is to make an attempt to generalise the model of pricing European options with an illiquid underlying asset considered by Rogers and Singh (2010). We assume that an investor's decisions have only a temporary effect on the price, which is proportional to the square of the change of the number of asset units in the investor's portfolio. We also assume that the underlying asset price follows a CEV model. To prove existence and uniqueness of the solution, we use techniques similar to fixed point theorems and Feynman-Kac representation. Asymptotic behaviour of the option price for small values of the illiquidity parameter is also analysed and a numerical procedure along with some numerical results is included.

1. Introduction. Most of the existing option pricing models do not take into account liquidity phenomena properly. The great impact of liquidity on pricing various securities was shown e.g. in [3], [17]. In the last decades numerous proposals for addressing this problem have appeared. Some of them assume a direct, permanent effect of the agent's actions on asset prices [16], 4], others include only temporary effects [21]. Most authors tend to agree that the average unit price of an asset for an investor is a convex function of the number of assets bought or sold, with minimum at 0 . Typically this function is assumed to be exponential [4] or quadratic [21] with good results. A second type function could be seen as the Taylor polynomial of degree two for a first type function [4]. The first type functions fit the data better, yet make calculations much harder. No matter which of these functions are used, even a short burst of great volatility will generate a huge

2010 Mathematics Subject Classification: Primary 60H30; Secondary 93E20.

Key words and phrases: liquidity, option pricing, hedging, price impact, CEV.

Received 17 September 2014; revised 14 January 2016.

Published online 21 April 2016. 
liquidity cost for a hedging investor. Thus, in option pricing with liquidity cost, modelling volatility is crucial.

In this paper we try to merge the model of liquidity cost proposed in [21] with the Constant Elasticity of Variance model (CEV for short, [6]). The latter is a generalisation of Black-Scholes, improved by allowing nonzero correlation between the observed price and volatility. In practise, price drops are connected with greater volatility in the nearest future, so that price and volatility are negatively correlated [5]. A popular name of this phenomena is the leverage effect. The CEV model, however, is used only in a short horizon.

Intuitively, price is not the only factor influencing volatility. Other factors vary over time. To cope with this problem, one may assume that the volatility is not constant, but e.g. follows the Black-Scholes model. The Wiener process used in the volatility model in general may be different from the one used in the CEV SDE, but it is usually assumed that both processes are simply correlated (i.e. $E\left(W_{1, t} W_{2, p}\right)=\rho t$ for all $t$, where $\rho$ is a constant correlation coefficient). This model is called (CEV) SABR (Stochastic Alpha Beta Rho) [11]. Currently, the concept of SABR commonly refers to a whole family of similarly designed models whose members may not use CEV. Whichever popular SABR model is used, calculations are laborious and in some places more advanced techniques are necessary due to an exogenous volatility process.

1.1. Outline. The paper is organised as follows. Subsection 1.2 briefly describes the CEV model. 1.3 contains all assumptions and motivation for choosing a particular cost function.

In Section 2 we state our optimisation problem and gradually transform it into a quasilinear PDE. In 2.1 we derive the Hamilton-Jacobi-Bellman equation. To simplify this equation, we calculate in 2.2 the optimal hedging strategy as a function of other unknown quantities appearing in this equation.

Finally in Section 3 we transform the problem into a system of three simpler PDEs. Subsection 3.1 contains a rather technical proof of integrability of our solution candidate. 3.2 states and proves the main result of this paper: existence of solutions for a specific class of quasilinear degenerate parabolic PDEs. 3.3 contains some final remarks and proof that the assumptions of the HJB theorem from 2.1 are satisfied.

Section 4 describes a numerical method inspired by the theoretical result of 3.2, as well as numerical results with some comments.

Section 5 is the derivation of some asymptotic properties, which contrary to earlier assessments ([21]) state that in our framework the cost of liquidity incurred at selling the whole portfolio near the option exercise date is an important factor. This section is rather technical. 
1.2. Constant Elasticity of Variance. In the CEV model, the asset price dynamics is given by the stochastic differential equation

$$
d S_{t}=\mu S_{t} d t+S_{t}^{1+\gamma} \sigma d W_{t}
$$

where $\mu$ and $\sigma$ play the same role as their counterparts in the Black-Scholes model. The parameter $\gamma$ describes how the volatility reacts on the low or high asset price.

LEMMA 1.1. The SDE (1.1) has a unique weak solution [7, which is a strong solution if one stops the process $X$ when it hits 0 (the natural boundary condition).

Proof. We can use [15, Chap. II, Th. 5.2] or [14, Ch. IV, Th. 3.1]. In the one-dimensional case they state that the SDE

$$
d X_{t}=a\left(X_{t}\right) d t+b\left(X_{t}\right) d W_{t},
$$

with $a$ and $b$ locally Lipschitz on a domain $D$, has a unique maximal solution up to explosion time (in particular $(s, t, x) \mapsto\left(X_{t} \mid X_{s}=x\right)$ is continuous). For $\mu=0, a(x)=0$ and $b(x)=\sigma x^{1+\gamma}$ on $D=\mathbb{R}_{+}$the assumptions are fulfilled.

Since the maximal solution on $D=\mathbb{R}_{+}$is unique, it must be the trace of the unique strong solution on $[0, \infty)$ (see [15]) with the condition $X_{t}=0 \Rightarrow$ $X_{u}=0$ for $u \geq t$ from [15, Chap. II, Th. 5.7] (Feller test in one dimension).

$E_{t, S}(\cdot)$ will denote conditional expectation with respect to $S_{t}=S$. It is well defined as an integrable process $\left(S_{s}\right)_{s>t}$ satisfying 1.1 with nonnegative initial condition $S_{t}=S$.

1.3. Assumptions and the cost function. Let us first say a few words about the CEV model. We will focus on the case of $\gamma<0$, since many pieces of empirical evidence [10, [2], 5] have shown that the correlation between the stock price and its return volatility is negative. With negative $\gamma$ the CEV model exhibits unpleasant properties like positive probability of bankruptcy in finite time or that the coefficients in the model SDE are not Lipschitz. We assume $\gamma \geq-1 / 2$ to avoid even more problems. Without loss of generality we can assume that $\mu=0$. This can be done by discounting data by $\mu$. The new $S_{t}$ is the old $S_{t} e^{-\mu t}$. The data processed in this manner will fit the $\mu=0$ case. This can be easily shown by applying Ito's lemma.

The basic assumptions are taken from [21. Assume that the liquidity cost is proportional to the asset price with constant $l(\cdot)\left(l: \mathbb{R} \rightarrow \mathbb{R}^{+}\right)$ depending only on the number $h$ of units sold or bought, i.e. the timederivative of the portfolio. For simplicity we assume that $l$ is a quadratic function, $l(h)=\epsilon h^{2} / 2$ for some constant $\epsilon$. As mentioned in the introduction, it fits data quite well [4]. We also assume that the price change connected with liquidity is temporary, and the market almost immediately returns to 
equilibrium, i.e. to $S_{t}$ before next transactions. Therefore, the cost (per unit time) of buying $h_{t}$ units (per unit time) at time $t$ at the market price $S_{t}$ is

$$
l\left(h_{t}\right) S_{t}+h_{t} S_{t} .
$$

Similarly, the total cash flow when following the (continuous-time) strategy $h_{t}, t \in\left[t_{0}, t_{1}\right]$, is

$$
\int_{t_{0}}^{t_{1}}\left(l\left(h_{t}\right)+h_{t}\right) S_{t} d t .
$$

This idea mimics the stock exchange mechanism, where every bid is processed as a set of bids on one share. Because every bid is matched with the best remaining opposite offer, our next bid will be matched with an opposite offer worse than the previous one. In formal language $l(\cdot)$ is strictly convex and it has exactly one minimum, at 0 . For simplicity we assume that $l(0)=0$ and $l(\cdot)$ is differentiable. This implies that $l^{\prime}(\cdot)$ is increasing.

The number of shares in the portfolio at time $t$ is denoted by $H_{t}$. Using the previous notation, we have

$$
H_{t}=H_{0}+\int_{0}^{t} h_{s} d s .
$$

To prove any meaningful results we must make some assumptions on $H$. We require

$$
\begin{aligned}
& E_{t, S, H}\left(\int_{t}^{T} H_{s}^{2} S_{s}^{2} d S_{s}\right)<\infty, \\
& E_{t, S, H}\left(\int_{0}^{T} l\left(h_{s}\right) S_{s} d s\right)<\infty .
\end{aligned}
$$

The first assumption ensures that the value of the portfolio $\left(H_{t} S_{t}\right)_{t \in[0, T]}$ is square integrable. The second assumption says that the portfolio should not be changed too quickly (because of the liquidity cost). Both conditions are quite natural. Hedging strategies in different classical models fulfil similar conditions. The set of all dynamics $\left(h_{t}\right)_{t \in[0, T]}$ of $\left(H_{t}\right)_{t \in[0, T]}$ which fulfil our assumptions will be called $\mathcal{H}$.

Let $x_{0}$ be an initial cash value. The classical (without the liquidity cost) value of the entire portfolio at time $t$ is denoted by $\xi_{t}$. It is given by

$$
\xi_{t}=H_{0} S_{0}+x_{0}+\int_{0}^{t} H_{s} d S_{s} .
$$

We want to hedge an option which pays $G\left(S_{T}\right)$ at time $T$. Its price $q(t, S)$ at time $t$ given the asset price $S$ is assumed to be the expected value of the 
payment at the end, and to be $C^{1,3}$ for $t<T$. It is also the value of the arbitrage strategy (without the liquidity cost). This relationship might be expressed as the partial differential equation

$$
\begin{gathered}
L(q)=0, \\
q(T, \cdot)=G(\cdot),
\end{gathered}
$$

where $L$ is the infinitesimal generator in $\mathrm{CEV}$ :

$$
L(\cdot)(t, S)=\lim _{r \downarrow 0} \frac{E_{t, S}\left(\cdot\left(t+r, S_{t+r}\right)-\cdot\left(t, S_{t}\right)\right)}{r} .
$$

For $C^{2}$ functions (Dynkin's formula [20, 7.4.1, (i)] is sufficient for our purposes),

$$
L(\cdot)=\frac{1}{2} \sigma^{2} S^{2+2 \gamma} \frac{\partial^{2} \cdot}{\partial S^{2}}+\frac{\partial \cdot}{\partial t} .
$$

We denote $\theta=\frac{\partial q}{\partial S}$, which is a self-financing strategy replicating the option (arbitrage) in the perfectly liquid environment (also known as the optimal pricing strategy). This will be the base for our strategy in an illiquid environment. In order to prove later results, we must assume that $\theta_{t}$ is bounded as follows:

$$
\exists_{C, \alpha>0} \forall_{S>0, t \in[0, T]}: \quad|\theta(t, S)|<C\left(1+S^{\alpha}\right) .
$$

A result from the last section of this paper (that $V$ is $o\left(\epsilon^{k}\right)$ ) requires an additional assumption:

$$
\exists_{C_{1}, \beta>0} \forall_{S>0, t \in[0, T]}: \quad\left|\frac{\partial \theta(t, S)}{\partial S}\right|<C\left(1+S^{\beta}\right) .
$$

After restriction to $t<T$ this condition holds for the standard testing object, European call/put options in the CEV model (see the formula for pricing European options in [6]). The only problem arises at $t=T, S=K$, where $q$ does not have a classical derivative with respect to $S$.

2. Value function and HJB equation. Clearly $\xi_{t}$ perfectly fitting the option's price is a rapidly changing strategy. This results in a huge cost of liquidity. To minimise that cost, we should pick a constant $h_{t} \equiv 0$. However, this strategy is pointless from the point of view of hedging. Therefore, we should minimise the mean squared error of hedging (neglecting illiquidity) under the condition that the mean liquidity cost stays reasonably low:

$$
\min _{\left(h_{t}\right): E_{t, S}\left(\int_{t}^{T} l\left(h_{s}\right) S_{s} d s\right) \leq C} \frac{1}{2} E_{t, S}\left(\left(G\left(S_{T}\right)-H_{T}\right)^{2}\right)
$$

where $H_{t}$ is our portfolio and $h_{t}$ is its dynamics $\left(d H_{t}=h_{t} d t\right)$ as in the previous section. To solve the above constrained optimization problem we 
cannot directly use stochastic HJB equations and therefore we first consider the Lagrange multiplier method and the problem

$$
\min _{\left(h_{t}\right)}\left(\frac{1}{2} E_{t, S, H}\left(\left(G\left(S_{T}\right)-\xi_{T}\right)^{2}\right)+\lambda E_{t, S, H}\left(\int_{t}^{T} l\left(h_{s}\right) S_{s} d s\right)\right) .
$$

Note that we could omit $\lambda$ by including it into the liquidity cost function $l$. Later $l(h)$ will be assumed to be of the form $\epsilon h^{2} / 2$ and $\lambda$ will be hidden in $\epsilon$. We will minimise

$$
\psi_{0}=\frac{1}{2} E_{t, S}\left(\left(G\left(S_{T}\right)-\xi_{T}\right)^{2}\right)+E_{t, S}\left(\int_{t}^{T} l\left(h_{s}\right) S_{s} d s\right) .
$$

We point out that our result from the last section says basically that for very low liquidity cost (small $\epsilon$ ) the cost of selling the portfolio at time $T$ cannot be neglected. Our market is not perfectly liquid, so we have to sell the final portfolio (or part of it) not instantly, but during some period of time $T^{\prime}$. Therefore, it will also be of interest to find the optimal strategy which minimises

$$
\bar{\psi}_{0}=\frac{1}{2} E_{t, S, H}\left(\left(G\left(S_{T}\right)-\xi_{T}\right)^{2}\right)+E_{t, S, H}\left(\int_{t}^{T} l\left(h_{s}\right) S_{s} d s+T^{\prime} l\left(\frac{H_{T}}{T^{\prime}}\right) S_{T}\right) .
$$

Note that $E\left(S_{T}\right)=S_{t}$, because $\mu=0$. Most of the further steps are the same for $\bar{\psi}_{0}$ and $\psi_{0}$. For this reason we will only give the proofs for $\psi_{0}$.

In this form the cost function is hard to handle, so we transform it using Ito's formula with $E_{t, S_{0}}\left(G\left(S_{T}\right)\right)=q\left(0, S_{0}\right)$ and then apply Ito's isometry to $E\left(\left(\int_{t}^{T}\left(\theta_{s}\left(S_{s}\right)-H_{s}\right) d S_{s}\right)^{2}\right)$ :

$$
\begin{aligned}
\psi_{0}= & \frac{1}{2}\left(x_{t}+H_{t} S_{t}-q_{0}\left(S_{t}\right)\right)^{2} \\
& +\frac{1}{2} E_{t, S_{t}, H_{t}}\left(\int_{t}^{T}\left(\theta\left(s, S_{s}\right)-H_{s}\right)^{2} \sigma^{2} S_{s}^{2+2 \gamma} d s\right)+E_{t, S_{t}, H_{t}}\left(\int_{t}^{T} S_{s} l\left(h_{s}\right) d s\right) \\
= & \frac{1}{2}\left(x_{t}+H_{t} S_{t}-q_{0}\left(S_{t}\right)\right)^{2}+\psi .
\end{aligned}
$$

We can omit the terms independent of the chosen strategy, as this does not change optimal strategies, so we will minimise just $\psi$. We point out that constants like $H_{0}$ still appear in $\psi$ in a hidden form, because $H_{t}=$ $H_{0}+\int_{0}^{t} h_{s} d s$ by 1.3 . Finally, our cost function $V(t, H, S)$ is given by

$$
\inf _{h \in \mathcal{H}} \frac{1}{2} E_{t, S, H}\left(\int_{t}^{T}\left(\theta\left(u, S_{u}\right)-H_{u}\right)^{2} \sigma^{2} S_{u}^{2+2 \gamma} d u+\int_{t}^{T} S_{u} l\left(h_{u}\right) d u\right),
$$


and similarly for 2.2 ,

$$
\inf _{h \in \mathcal{H}} \frac{1}{2} E_{t, S, H}\left(\int_{t}^{T}\left(\theta\left(u, S_{u}\right)-H_{u}\right)^{2} \sigma^{2} S_{u}^{2+2 \gamma} d u+\int_{t}^{T} S_{u} l\left(h_{u}\right) d u+\frac{\epsilon S_{T} H_{T}^{2}}{2 T^{\prime}}\right) .
$$

2.1. HJB equation. We shall use a modification of [20, Th. 11.2.2] (the latter theorem concerns a maximization problem).

Lemma 2.1. Suppose that:

(1) $S_{t}$ follows the CEV model (1.1).

(2) $q(t, S)$ is the price of the option which pays $G\left(S_{T}\right)$ at time $T$.

(3) $q(t, S)$ is $C^{1,3}$ for $t<T$ and $\frac{\partial q}{\partial S}=\theta$ satisfies (1.7).

Moreover, suppose that a solution $V$ of the HJB equation

$$
0=\min _{h}\left(h V_{H}+L(V)+\frac{1}{2} \sigma^{2} S^{2+2 \gamma}(\theta(t, S)-H)^{2}+S l(h)\right)
$$

with the terminal condition $V(T, H, S)=0$ is of class $C^{2}$ and $V\left(t, H_{t}, S_{t}\right)$ is uniformly integrable for all controls $\left(h_{s}\right)_{t \leq s \leq T}$ satisfying (1.4). Then $V$ is the minimal value of the cost function

$$
\frac{1}{2} E_{t, S, H}\left(\int_{t}^{T}\left(\theta\left(u, S_{u}\right)-H_{u}\right)^{2} \sigma^{2} S_{u}^{2+2 \gamma} d u+\int_{t}^{T} S_{u} l\left(h_{u}\right) d u\right) .
$$

Recall that $L$ (see (1.6)) is the CEV infinitesimal generator.

Proof. The only problem is to calculate the infinitesimal generator $M$ of $Y_{t}=\left(t, S_{t}, H_{t}\right)$. Let us recall the dynamics of the ingredients:

$$
d(t)=d t, \quad d\left(S_{t}\right)=\sigma S_{t}^{1+\gamma} d W_{t}, \quad d\left(H_{t}\right)=h_{t} d t .
$$

By [20, Th. 7.3.3],

$$
M(f)=h_{t} \frac{\partial f}{\partial H}+L(f) .
$$

We apply [20, Th. 11.2.2] with the technical change stated before to process $Y_{t}$ with control $h_{t}$ in order to find an optimal strategy for our problem (minimising the value of (2.1)). Suppose that $V$ is $C^{1,2}$ and it is the solution of the PDE

$$
0=\inf _{h}\left(V_{t}+h V_{H}+\frac{1}{2} \sigma^{2} S^{2+2 \gamma} V_{S S}+\frac{1}{2} \sigma^{2} S^{2+2 \gamma}(\theta(t, S)-H)^{2}+S l(h)\right)
$$

with the terminal condition $V(T, H, S)=0$, due to the absence of $T, S_{T}, H_{T}$ dependent term in the cost function. Assume that $h$ at which the minimum is attained exists at every point and $V\left(t, H_{t}, S_{t}\right)$ is uniformly integrable. Then according to [20, Th. 11.2.2], $V$ is the true value function, and the optimal control $\left(h_{t}\right)_{t \in[0, T]}$ can be found by calculating the argument of the minimum in 2.6 . 
To simplify notation we will use the operator $L$, defined in (1.6):

$$
0=\inf _{h}\left(h V_{H}+L(V)+\frac{1}{2} \sigma^{2} S^{2+2 \gamma}(\theta(t, S)-H)^{2}+S l(h)\right) .
$$

$h$ is still a function of time, as $V$ is a function of time, of the initial asset price, and of the initial number of asset units. For simplicity we omit all these variables everywhere we can. We then notice that stochasticity has disappeared and we are dealing with a deterministic partial differential equation.

For the case with the cost of liquidating the portfolio at the end, the proof is similar and we omit it.

LEMMA 2.2. Suppose that:

(1) $S_{t}$ follows the CEV model (1.1).

(2) $q(t, S)$ is the price of the option which pays $G\left(S_{T}\right)$ at time $T$.

(3) $q(t, S)$ is $C^{1,3}$ for $t<T$ and $\frac{\partial q}{\partial S}=\theta$ satisfies 1.7 .

Moreover, suppose that a solution $\bar{V}$ of the PDE

$$
0=\min _{h}\left(h \bar{V}_{H}+L(\bar{V})+\frac{1}{2} \sigma^{2} S^{2+2 \gamma}(\theta(t, S)-H)^{2}+S l(h)\right)
$$

with the terminal condition $\bar{V}(T, H, S)=T^{\prime} l\left(\frac{H}{T^{\prime}}\right) S$ is of class $C^{2}$ and $\bar{V}\left(t, S_{t}, H_{t}\right)$ is uniformly integrable for all controls $\left(h_{s}\right)_{t \leq s \leq T}$ satisfying (1.4). Then $\bar{V}$ is the minimal value of the cost function

$$
\begin{aligned}
\inf _{h \in \mathcal{H}} \frac{1}{2} E_{t, S, H}\left(\int_{t}^{T}\left(\theta\left(u, S_{u}\right)-H_{u}\right)^{2} \sigma^{2}\right. & S_{u}^{2+2 \gamma} d u \\
& \left.+\int_{t}^{T} S_{u} l\left(h_{u}\right) d u+T^{\prime} l\left(\frac{H_{T}}{T^{\prime}}\right) S_{T}\right) .
\end{aligned}
$$

It is possible to continue with a general $l(\cdot)$, but then a convex conjugate appears in a partial differential equation, which cannot be handled easily. For this reason we pick a simple strictly convex $l(\cdot)$ with a simple convex conjugate. Now we will try to calculate it as a function of $S, H, t$ and of values of $V$. This will allow us to substitute $h$ in the equation above and, as a result, we will get some PDE in a standard form to solve.

2.2. Calculating $h$ from (2.5). Equation (2.5) involves only two terms depending on $h: S l(h)$ and $h V_{H}$. Without any assumptions on $h$ it can be calculated as the infimum over $S, H, t, V$ :

$$
h=\underset{\tilde{h}}{\arg \min }\left(S l(\tilde{h})+\tilde{h} V_{H}\right) .
$$

Denote the convex conjugate of $-S l$ as $(-S l)^{*}$. Then (2.5) takes the form

$$
0=L(V)+\frac{1}{2} \sigma^{2} S^{2+2 \gamma}(\theta-H)^{2}+(-S l)^{*}\left(V_{H}\right) .
$$


Later results require choosing some particular form of $l$. We pick $l(h)=\epsilon h^{2} / 2$ as mentioned in Subsection 1.3 . Then $(-S l)^{*}(\cdot)=(\cdot)^{2} /(2 S \epsilon)$.

Lemma 2.3. For a given solution $V$ of

$$
\begin{aligned}
& 0=\inf _{h}\left(h V_{H}+L(V)+\frac{1}{2} \sigma^{2} S^{2+2 \gamma}(\theta(t, S)-H)^{2}+S l(h)\right), \\
& \forall_{S, H}: V(T, H, S)=0
\end{aligned}
$$

with $l(h)=\epsilon h^{2} / 2$ the optimal $h$ exists and is given by

$$
h=-\frac{V_{H}}{S \epsilon} .
$$

Moreover, $V$ is a solution of the above-mentioned PDE if and only if it is a solution of

$$
0=L(V)+\frac{1}{2}\left(\sigma^{2} S^{2+2 \gamma}(\theta-H)^{2}-\frac{V_{H}^{2}}{2 S \epsilon}\right) .
$$

Proof. To calculate the infimum (2.5), we will use the necessary condition (zero first derivative). Since $l$ is also strictly convex and $h V_{H}$ is a convex function of $h$, their sum is strictly convex, and this condition is also sufficient. We get $S l^{\prime}(h)+V_{H}=0$, so

$$
l^{\prime}(h)=-\frac{V_{H}}{S} .
$$

In our case $l^{\prime}(h)=\epsilon h$. Since both $S$ and $\epsilon$ cannot be 0 ,

$$
h=-\frac{V_{H}}{S \epsilon} \text {. }
$$

Substituting this into 2.5, we obtain the desired result:

$$
0=L(V)+\frac{1}{2}\left(\sigma^{2} S^{2+2 \gamma}(\theta-H)^{2}-\frac{V_{H}^{2}}{2 S \epsilon}\right)
$$

The same proof holds for the case with the cost of liquidating the portfolio at the end.

Lemma 2.4. For a given solution $V$ of

$$
\begin{aligned}
& 0=\inf _{h}\left(h \bar{V}_{H}+L(\bar{V})+\frac{1}{2} \sigma^{2} S^{2+2 \gamma}(\theta(t, S)-H)^{2}+S l(h)\right), \\
& \forall_{S, H}: \quad \bar{V}(T, H, S)=\frac{\epsilon S H^{2}}{2 T^{\prime}}
\end{aligned}
$$

with $l(h)=\epsilon h^{2} / 2$ the optimal $h$ exists and is given by

$$
h=-\frac{V_{H}}{S \epsilon} .
$$


Moreover, $V$ is a solution of the above-mentioned PDE if and only if it is a solution of

$$
0=L(V)+\frac{1}{2}\left(\sigma^{2} S^{2+2 \gamma}(\theta-H)^{2}-\frac{V_{H}^{2}}{2 S \epsilon}\right)
$$

3. Derivation and solution of the final PDE. We notice the appearance of terms quadratically depending on $V_{H}$ and linearly on other derivatives. This suggests that $V$ might depend only quadratically on $H$. This supposition will lead us to (exactly) one solution. We have:

LEMMA 3.1. If we have a $C^{1,2}$ solution $(a, b, c)$ of the system of PDEs

$$
\begin{aligned}
& 0=L(a)+\frac{1}{2} \sigma^{2} S^{2+2 \gamma}-\frac{2 a^{2}}{S \epsilon}, \\
& 0=L(b)-\sigma^{2} S^{2+2 \gamma} \theta-\frac{2 a b}{S \epsilon}, \\
& 0=L(c)+\frac{1}{2} \sigma^{2} S^{2+2 \gamma} \theta^{2}-\frac{b^{2}}{2 S \epsilon},
\end{aligned}
$$

with the terminal condition

$$
\forall_{S}: \quad a(T, S)=b(T, S)=c(T, S)=0,
$$

then

$$
V(t, H, S)=a(t, S) H^{2}+b(t, S) H+c(t, S)
$$

is a solution of the main PDE 2.11.

Proof. Suppose $V(t, H, S)$ is as in 3.5 for some functions $a, b, c$ that are $C^{2}$ in $S$ and $C^{1}$ in $t$. Now, equation 2.11 takes the form

$$
0=L\left(a H^{2}+b H+c\right)+\frac{1}{2}\left(\sigma^{2} S^{2+2 \gamma}(\theta-H)^{2}-\frac{(2 a H+b)^{2}}{2 S \epsilon}\right),
$$

that is,

$$
\begin{aligned}
0= & L(a) H^{2}+L(b) H+L(c) \\
& +\frac{1}{2}\left(\sigma^{2} S^{2+2 \gamma}\left(\theta^{2}+2 \theta H+H^{2}\right)-\frac{4 a^{2} H^{2}+4 a b H+b^{2}}{2 S \epsilon}\right) .
\end{aligned}
$$

By comparing the coefficients of different powers of $H$ we get the system (3.1) - 3.3. The terminal condition $V(T, H, S)=0$ for all $H, S$ follows immediately from 3.4 .

The version for the case with the cost of liquidation of the final portfolio is as follows: 
LEMma 3.2. If we have a $C^{1,2}$ solution $(\bar{a}, \bar{b}, \bar{c})$ of the system of PDEs

$$
\begin{aligned}
& 0=L(\bar{a})+\frac{1}{2} \sigma^{2} S^{2+2 \gamma}-\frac{2 \bar{a}^{2}}{S \epsilon}, \\
& 0=L(\bar{b})-\sigma^{2} S^{2+2 \gamma} \theta-\frac{2 \bar{a} \bar{b}}{S \epsilon}, \\
& 0=L(\bar{c})+\frac{1}{2} \sigma^{2} S^{2+2 \gamma} \theta^{2}-\frac{\bar{b}^{2}}{2 S \epsilon},
\end{aligned}
$$

with the terminal condition

$$
\forall_{S}: \quad \bar{a}(T, S)=\frac{\epsilon S}{2 T^{\prime}}, \quad \bar{b}(T, S)=\bar{c}(T, S)=0,
$$

then

$$
\bar{V}(t, H, S)=\bar{a}(t, S) H^{2}+\bar{b}(t, S) H+\bar{c}(t, S)
$$

is a solution of the PDE (2.16).

If we have a solution $a$ of (3.1), equation 3.2 will be easy to solve by using a technique analogous to Feynman-Kac representation. Similarly we would proceed with 3.3 by using $b$ in the same way as $a$ before.

The term $a^{2}$ is rather problematic to deal with. For this reason, one $a$ will be considered as known and the other as unknown. This reasoning leads us to an auxiliary PDE:

LEMmA 3.3. If for a given $C^{2}$ function $\widehat{a}$, a function $\widetilde{a}$ is a solution of

$$
0=L(\widetilde{a})+\frac{1}{2} \sigma^{2} S^{2+2 \gamma}-\frac{2 \widetilde{a} \widehat{a}}{S \epsilon}
$$

with the terminal condition $\forall_{S}: \widetilde{a}(T, S)=0$, and if $\widehat{a}=\widetilde{a}$, then $a=\widehat{a}$ solves the PDE (3.1).

Proof. If we set $\widehat{a}=\widetilde{a}$ into 3.10 , we get (3.1).

The same proof works in the second case.

LEMmA 3.4. If for a given $C^{2}$ function $\widehat{a}$, a function $\widetilde{a}$ is a solution of

$$
0=L(\widetilde{a})+\frac{1}{2} \sigma^{2} S^{2+2 \gamma}-\frac{2 \widetilde{a} \widehat{a}}{S \epsilon}
$$

with the terminal condition

$$
\forall_{S}: \quad \bar{a}(T, S)=\frac{\epsilon S}{2 T^{\prime}}, \quad \bar{b}(T, S)=\bar{c}(T, S)=0,
$$

and if $\widehat{a}=\widetilde{a}$, then $\bar{a}=\widehat{a}$ solves the PDE (3.6).

3.1. Integrability of an analogue of Feynman-Kac representation for the auxiliary PDE. To solve (3.10) we will use a reasoning similar to Feynman-Kac representation. For $\gamma>0$ we can use the version of Feynman-Kac representation from [12] as explained in detail for CEV in Subsection 2.3. For $\gamma<0$ we cannot use it directly, because $S^{1+\gamma}$ is not 
locally Lipschitz on the range of values attainable by the process $S_{t}$ (in this case $[0, \infty)$ ). We will calculate the solution as in the Feynman-Kac formula, but we will prove its properties directly.

Lemma 3.5. The following inequality holds for $C E V S_{t}$ :

$$
E_{t, S}\left(S_{u}^{2+2 \gamma}\right) \leq \widehat{C}\left(S_{t}^{2+2 \gamma}+1\right)\left((T-u)^{1+2 \gamma}+1\right)
$$

for $t \leq u \leq T$ and some constant $\widehat{C}$ depending only on $\gamma, \sigma$. Additionally $(t, u, S) \mapsto\left(S_{u} \mid S_{t}=S\right)$ is continuous.

Proof. The integral $E_{t, s}\left(S_{u}^{2+2 \gamma}\right)$ is well defined, possibly infinite. Since $S_{u}$ is a CEV process, it has a known density [22], [13]. We use the notation of [13] (only $\gamma$ will remain unchanged and $r=a=0$ ):

$$
k=\frac{1}{\sigma^{2} 4 \gamma^{2}(u-t)}, \quad x=k S_{t}^{-2 \gamma}, \quad z=k S_{u}^{-2 \gamma} .
$$

Also, we change the variable

$$
d S_{u}=(-2 \gamma)^{-1} k^{\frac{1}{-2 \gamma}} w^{\frac{1+2 \gamma}{-2 \gamma}} d z
$$

in the density

$$
\begin{aligned}
& f\left(S_{u} \mid S_{t}, u>t\right) \\
= & \int_{0}^{\infty} 2 \gamma k^{\frac{1}{-2 \gamma}}\left(x z^{1-2 \gamma}\right)^{\frac{1}{-4 \gamma}} \exp (-x-z) I_{\frac{1}{-2 \gamma}}(2 \sqrt{x z})(-2 \gamma)^{-1} k^{\frac{1}{-2 \gamma}} w^{\frac{1+2 \gamma}{-2 \gamma}} d z .
\end{aligned}
$$

Now we can transform $E_{t, S}\left(S_{u}^{2+2 \gamma}\right)$ into

$$
\begin{aligned}
\int_{0}^{\infty}\left(\frac{z}{k}\right)^{\frac{2+2 \gamma}{-2 \gamma}} 2 \gamma k^{\frac{1}{-2 \gamma}} & \left(x z^{1-2 \gamma}\right)^{\frac{1}{-4 \gamma}} \exp (-x-z) I_{\frac{1}{-2 \gamma}}(2 \sqrt{x z})(-2 \gamma)^{-1} k^{\frac{1}{-2 \gamma}} w^{\frac{1+2 \gamma}{-2 \gamma}} d z \\
= & \int_{0}^{\infty}\left(\frac{z}{k}\right)^{\frac{2+2 \gamma}{-2 \gamma}} 2 \gamma\left(\frac{x}{z}\right)^{\frac{1}{-4 \gamma}} \exp (-x-z) I_{\frac{1}{-2 \gamma}} 2 \sqrt{x z} d z \\
= & \left(\frac{x}{k}\right)^{\frac{1}{-2 \gamma}} \int_{0}^{\infty} z^{\frac{1+2 \gamma}{-2 \gamma}} 2 \gamma\left(\frac{z}{x}\right)^{\frac{1}{-4 \gamma}} \exp (-x-z) I_{\frac{1}{-2 \gamma}}(2 \sqrt{x z}) d z .
\end{aligned}
$$

Such transformations are used in the detailed calculation of the CEV European pricing formula in [13] (more precisely the $C_{1}$ term, from the beginning to page 22). This integral is the $\frac{1+2 \gamma}{-2 \gamma}=\frac{1}{-2 \gamma}-1$ moment of the chi square distribution with $2-1 / \gamma$ degrees of freedom and with noncentrality parameter $2 x$. The moment generating function of the noncentral chi square distribution is

$$
M(t ; d f, n c p)=\frac{\exp \left(\frac{n c p t}{1-2 t}\right)}{(1-2 t)^{k / 2}} .
$$


The $n$th moment is the $n$th derivative of $M$ at $t=0$, multiplied by a known constant. At $t=0$ we have $1-2 t=1$ and $\exp \left(\frac{n c p t}{1-2 t}\right)=1$. Thus, the $n$th moment is a polynomial of order $n$ in the variables $d f$ and $n c p$. The coefficients only depend on $\gamma, \sigma$, and $n$. If $\frac{1}{-2 \gamma}-1$ is not an integer, we can use some larger integer $r>\frac{1}{-2 \gamma}-1$ and the Hölder inequality to obtain

$$
E\left(X^{r}\right) \leq E\left(X^{\frac{1}{-2 \gamma}-1}\right)^{\frac{r}{-2 \gamma}-1} .
$$

Therefore, without loss of generality we can assume that $\frac{1}{-2 \gamma}-1$ is an integer. Every polynomial of degree $n$ can be majorized by some constant $C$ (e.g. the sum of the absolute values of the coefficients) multiplied by the absolute value of the highest order term $|x|^{n}$ plus 1 . In our case $x$ is nonnegative and the absolute value can be dropped. We will gradually return to our old notation. For $\frac{1}{-2 \gamma}-1$ an integer we get

$$
E_{t, S}\left(S_{u}^{2+2 \gamma}\right) \leq C(x / k)^{\frac{1}{-2 \gamma}}\left(x^{\frac{1}{-2 \gamma}-1}+1\right),
$$

so

$$
E_{t, S}\left(S_{u}^{2+2 \gamma}\right) \leq C\left(S_{t}^{-2 \gamma}\right)^{\frac{1}{-2 \gamma}}\left(\left(k S_{t}^{-2 \gamma}\right)^{\frac{1}{-2 \gamma}-1}+1\right) .
$$

Recall that $k$ depends on time, which is important from our perspective. Terms like $\gamma, \sigma$ are added to the new constant $\widehat{C}$ in

$$
E_{t, S}\left(S_{u}^{2+2 \gamma}\right) \leq \widehat{C}\left(S_{t}^{2+2 \gamma}+1\right)\left((T-u)^{1+2 \gamma}+1\right)
$$

CEV (Lemma 1.1) is a strong solution (in the sense of [15] and [14, Ch. IV, Def. 1.6]) and therefore $(t, u, S) \mapsto\left(S_{u} \mid S_{t}=S\right)(\omega)$ is continuous.

CEV also satisfies $\left(S_{t} \mid S_{s}=0\right)=0$ for $s<t$. We have already proved that $E_{t, S}\left(\int_{t}^{s} \sigma^{2} S_{u}^{2+2 \gamma} d u\right)$ is finite (see $(3.14)$ ), which is required for Ito isometry:

$$
\begin{aligned}
E_{t, S}\left(\int_{t}^{s} \sigma^{2} S_{u}^{2+2 \gamma} d u\right) & =E_{t, S}\left(\left(\int_{t}^{s} \sigma^{2} S_{u}^{2+2 \gamma} d W_{u}\right)^{2}\right) \\
& =E_{t, S}\left(\left(\int_{t}^{s} d S_{u}\right)^{2}\right)=E_{t, S}\left(\left(S_{s}-S_{t}\right)^{2}\right) .
\end{aligned}
$$

Again using (3.14) we get

$$
E_{t, S}\left(\left(S_{s}-S_{t}\right)^{2}\right) \leq C\left(S_{t}^{-2 \gamma}\right)^{\frac{1}{-2 \gamma}}\left(\left(k S_{t}^{-2 \gamma}\right)^{\frac{1}{-2 \gamma}-1}+1\right) .
$$

We know that always $S_{t} \geq 0$, thus as $S \downarrow 0,\left(S_{s} \mid S_{t}=S\right)$ converges to a constant 0 . We have shown earlier that $(s, t, x) \mapsto\left(X_{t} \mid X_{s}=x\right)$ is continuous on $(0, \infty)^{2} \times(0, \infty)$ and on $(0, \infty)^{2} \times\{0\}$; this combined with continuity at 0 gives continuity in the whole $[0, \infty)$. 
LEMma 3.6. For a given nonnegative continuous function $\widehat{a}$ the function $\widetilde{a}$ defined as

$$
\widetilde{a}(t, S)=\frac{1}{2} E_{t, S}\left(\int_{t}^{T} e^{-\int_{t}^{u} 2 \widehat{a}\left(\nu, S_{\nu}\right) \frac{d \nu}{\epsilon S_{\nu}}} \sigma^{2} S_{u}^{2+2 \gamma} d u\right)
$$

is well defined, nonnegative and continuous.

Proof. Since $e^{-\int_{t}^{u} 2 \widehat{a}\left(\nu, S_{\nu}\right) \frac{d \nu}{\epsilon S_{\nu}}}$ and $\sigma^{2} S_{u}^{2+2 \gamma}$ are measurable and nonnegative, $\widetilde{a}$ is also nonnegative (possibly infinite).

We must show that the right side above is finite. Because the exponent is always nonpositive, the whole exponential term is smaller than 1 . Hence

$$
\begin{aligned}
\frac{1}{2}\left(\int_{t}^{T} e^{-\int_{t}^{u} 2 \widehat{a}\left(\nu, S_{\nu}\right) \frac{d \nu}{\epsilon S_{\nu}}} \sigma^{2} S_{u}^{2+2 \gamma} d u \mid S_{t}\right. & =S) \\
& \leq \frac{1}{2}\left(\int_{t}^{T} \sigma^{2} S_{u}^{2+2 \gamma} d u \mid S_{t}=S\right) .
\end{aligned}
$$

The right side above is integrable according to Lemma 3.5. We can use Tonelli's theorem [1, Th. 5.2.1] (for a $\sigma$-finite measure) to interchange the expected value and Lebesgue integral (if the resulting expression is finite):

$$
\frac{1}{2} E_{t, S}\left(\int_{t}^{T} \sigma^{2} S_{u}^{2+2 \gamma} d u\right) \stackrel{?}{=} \frac{1}{2} \int_{t}^{T} \sigma^{2} E_{t, S}\left(S_{u}^{2+2 \gamma}\right) d u .
$$

We can use Lemma 3.5 .

$$
\begin{aligned}
& \frac{1}{2} \int_{t}^{T} E_{t, S}\left(\sigma^{2} S_{u}^{2+2 \gamma}\right) d u \\
& \quad \leq \frac{1}{2} \sigma^{2} \widehat{C}\left(S_{t}^{2+2 \gamma}+1\right)\left(\frac{(T-t)^{2+2 \gamma}}{2+2 \gamma}-\frac{(T-T)^{2+2 \gamma}}{2+2 \gamma}+T-t\right) .
\end{aligned}
$$

Since the right side is finite (polynomials are easily integrated), the assumptions of Tonelli's theorem are fulfilled. By Tonelli's theorem the function $\frac{1}{2} E_{t, S}\left(\int_{t}^{T} \sigma^{2} S_{u}^{2+2 \gamma} d u\right)$ is also locally integrable. Because $\widetilde{a}$ is defined by an integral with a nonnegative, continuous integrand, and this integral is finite, $\widetilde{a}$ is well defined and locally integrable.

We recall the definition of $\widetilde{a}$ :

$$
\widetilde{a}(t, S)=\frac{1}{2} E_{t, S}\left(\int_{t}^{T} e^{-\int_{t}^{u} 2 \widehat{a}\left(\nu, S_{\nu}\right) \frac{d \nu}{\epsilon S_{\nu}}} \sigma^{2} S_{u}^{2+2 \gamma} d u\right) .
$$

From Lemma 3.5. $(t, s, S) \mapsto\left(S_{s} \mid S_{t}=S\right)$ is continuous. If $\widehat{a}$ is continuous, both $(t, \nu, S) \rightarrow\left(2 \widehat{a}\left(\nu, S_{\nu}\right) \frac{1}{\epsilon S_{\nu}} \mid S_{t}+S\right)$ and $(t, u, S) \rightarrow\left(\sigma^{2} S_{u}^{2+2 \gamma} \mid S_{t}+S\right)$ 
are continuous. The function given by an integral over a finite interval of a continuous function is also continuous. From 3.16,

$$
\frac{1}{2}\left(\int_{t}^{T} e^{-\int_{t}^{u} 2 \widehat{a}\left(\nu, S_{\nu}\right) \frac{d \nu}{\epsilon S_{\nu}}} \sigma^{2} S_{u}^{2+2 \gamma} d u \mid S_{t}=S\right) \leq \frac{1}{2}\left(\int_{t}^{T} \sigma^{2} S_{u}^{2+2 \gamma} d u \mid S_{t}=S\right) .
$$

Since $\gamma<0, g(x)=x^{\frac{2}{2+2 \gamma}}$ is convex for nonnegative numbers. Hence

$$
\left(\frac{1}{2}\left(\int_{t}^{T} e^{-\int_{t}^{u} 2 \widehat{a}\left(\nu, S_{\nu}\right) \frac{d \nu}{\epsilon S_{\nu}}} \sigma^{2} S_{u}^{2+2 \gamma} d u \mid S_{t}=S\right)\right)^{\frac{1}{1+\gamma}} \leq \frac{1}{2}\left(\int_{t}^{T} \sigma^{2} S_{u}^{2} d u \mid S_{t}=S\right) .
$$

We take expectations, then substitute (3.15) and use Tonelli's theorem to get

$$
\begin{aligned}
E_{t, S}\left(\frac{1}{2}\left(\int_{t}^{T} e^{-\int_{t}^{u} 2 \widehat{a}\left(\nu, S_{\nu}\right) \frac{d \nu}{\epsilon S_{\nu}}} \sigma^{2} S_{u}^{2+2 \gamma} d u\right)\right)^{\frac{1}{1+\gamma}} & \\
& \leq E_{t, S}\left(\frac{1}{2}\left(\int_{t}^{T} \widehat{C}\left(S^{2+2 \gamma}+1\right)\left((s-t)^{1+2 \gamma}+1\right) d s\right)\right) .
\end{aligned}
$$

On the right side, the term under the expectation sign continuously depends on $t \in\left[t_{0}-\epsilon, t_{0}+\epsilon\right]$ and $S \in\left[S_{0}-\epsilon, S_{0}+\epsilon\right]$, so the right side has a maximum at some $\left(t_{1}, S_{1}\right)$. By the de la Vallée-Poussin theorem ([1, Prop. 2.5.7(ii) or more general Remark 2.5.7], [18, 2.2 Th. 22]) with $g(x)=x^{\frac{2}{2+2 \gamma}}$, we have uniform integrability of the family

$$
\left(\int_{t}^{T} e^{-\int_{t}^{u} 2 \widehat{a}\left(\nu, S_{\nu}\right) \frac{d \nu}{\epsilon S_{\nu}}} \sigma^{2} S_{u}^{2+2 \gamma} d u \mid S_{t}=S\right)_{t \in\left[t_{0}-\epsilon, t_{0}+\epsilon\right], S \in\left[S_{0}-\epsilon, S_{0}+\epsilon\right]},
$$

i.e. terms like under the expectation sign in (3.18). Using the Dunford-Pettis theorem [18, 2.2 Th. 25], we see that for any sequences $S_{n} \rightarrow S$ and $t_{n} \rightarrow t$ such that $\left(t_{n}, S_{n}\right)_{n \in \mathbb{N}} \subset\left[t_{0}-\epsilon, t_{0}+\epsilon\right] \times\left[S_{0}-\epsilon, S_{0}+\epsilon\right]$ the values of $\widetilde{a}\left(t_{n}, S_{n}\right)$ converge to $\widetilde{a}(t, S)$. The choice of $\left[t_{0}-\epsilon, t_{0}+\epsilon\right] \times\left[S_{0}-\epsilon, S_{0}+\epsilon\right]$ was arbitrary and sets of this form cover the whole $[0, \infty)^{2}$. This gives the continuity of $\widetilde{a}$ wherever it is defined.

LEMMA 3.7.

$$
\widetilde{a}(t, S)=\frac{1}{2} E_{t, S}\left(\int_{t}^{T} e^{-\int_{t}^{u} 2 \widehat{a}\left(\nu, S_{\nu}\right) \frac{d \nu}{\epsilon S_{\nu}}} \sigma^{2} S_{u}^{2+2 \gamma} d u+\frac{\epsilon S_{T}}{2 T^{\prime}} e^{-\int_{t}^{T} 2 \widehat{a}\left(\nu, S_{\nu}\right) \frac{d \nu}{\epsilon S_{\nu}}}\right)
$$

is nonnegative for every integrable $\widehat{a}$ (possibly infinite). If additionally $\widehat{a}$ is nonnegative and continuous, then the above function is well defined, continuous and integrable.

The proof is analogous to the previous one. 
3.2. Solving the PDE for $a, b$ and $c$. We check if the following (integrable) function is, indeed, a solution of the auxiliary PDE.

LEMMA 3.8. If $\widehat{a}$ is continuous and nonnegative then

$$
\widetilde{a}(t, S)=\frac{1}{2} E_{t, S}\left(\int_{t}^{T} e^{-\int_{t}^{u} 2 \widehat{a}\left(\nu, S_{\nu}\right) \frac{d \nu}{\epsilon S_{\nu}}} \sigma^{2} S_{u}^{2+2 \gamma} d u\right)
$$

solves the auxiliary equation 3.10).

Proof. Since $\widetilde{a}(t, S)$ is nonnegative, integrable, we can use Tonelli's theorem:

$$
\widetilde{a}(t, S)=\frac{1}{2} \int_{t}^{T} E_{t, S}\left(e^{-\int_{t}^{u} 2 \widehat{a}\left(\nu, S_{\nu}\right) \frac{d \nu}{\epsilon S_{\nu}}} \sigma^{2} S_{u}^{2+2 \gamma}\right) d u .
$$

CEV is a pathwise unique strong solution (Lemma 1.1) of SDE (see 14, Def. IV.1.4]) with coefficients independent of $t$, hence $\left(S_{t+s} \mid S_{s}=S\right)_{t>0}$ is CEV in the filtration $\left(\mathcal{F}_{t+s}\right)_{t \geq 0}$ and hence has the following property: for every $S_{t}$-integrable $f$ (that is, $\forall_{t \leq u, x \geq 0}: E_{t, x}\left(\left|f\left(S_{u}\right)\right|\right)<\infty$ ) and any $T-u \leq h \leq 0$, we have $E_{t, x}\left(f\left(S_{u}\right)\right)=E_{t+h, x}\left(f\left(S_{u+h}\right)\right)$. One can find an integral formula for $E_{t, x}\left(f\left(S_{u}\right)\right)$ depending only on $u-t$ and $x$ using the density (3.13) given in the proof of Lemma 3.5. This fact allows us to transform (3.19) into

$$
\widetilde{a}(t, S)=\frac{1}{2} \int_{t}^{T} E_{t, S}\left(e^{-\int_{t}^{u} 2 \widehat{a}\left(\nu, S_{\nu-t}\right) \frac{d \nu}{\epsilon S_{\nu-t}}} \sigma^{2} S_{u-t}^{2+2 \gamma}\right) d u .
$$

The expected value of a process with continuous trajectories is continuous. As a function given by an integral with a continuous integrand, $\widehat{a}$ is differentiable with respect to $t$.

Let us define two adapted processes

$$
X_{t}=e^{\int_{t}^{T} 2 \widehat{a}\left(\nu, S_{\nu}\right) \frac{d \nu}{\epsilon S_{\nu}}}, \quad Z_{t}=\frac{1}{2} \int_{t}^{T} e^{-\int_{t}^{u} 2 \widehat{a}\left(\nu, S_{\nu}\right) \frac{d \nu}{\epsilon S_{\nu}}} \sigma^{2} S_{u}^{2+2 \gamma} d u
$$

with continuous trajectories. Then $Z_{t}$ is uniformly bounded: $0 \leq Z_{t} \leq$ $\frac{1}{2} \int_{t}^{T} \sigma^{2} S_{u}^{2+2 \gamma} d u$, by a square integrable random variable (Lemma 3.5 . The same applies to $Z_{t} X_{t}$ (same bound) and $1 / X_{t}(\leq 1)$ (for more details see below). This will allow us to calculate their stochastic derivatives using the Ito lemma and the stochastic derivative of the product.

Let us calculate

$$
Z_{t} X_{t}=\frac{1}{2} \int_{t}^{T} e^{\int_{u}^{T} 2 \widehat{a}\left(\nu, S_{\nu}\right) \frac{d \nu}{\epsilon S_{\nu}}} \sigma^{2} S_{u}^{2+2 \gamma} d u
$$

All terms on the right hand side are continuous (see proof of Lemma 3.6), so integrals over compact intervals are well defined. As $Z_{t} X_{t}$ is square inte- 
grable, we can use the Ito lemma to calculate the derivative:

$$
d\left(Z_{u} X_{u}\right)=-\frac{1}{2} e^{\int_{u}^{T} 2 \widehat{a}\left(\nu, S_{\nu}\right) \frac{d \nu}{\epsilon S_{\nu}}} \sigma^{2} S_{u}^{2+2 \gamma} d u .
$$

Since $Z_{t}=\left(Z_{t} X_{t}\right) \frac{1}{X_{t}}$, proceeding with $\frac{1}{X_{t}}$ as with $Z_{t} X_{t}$ before we get

$$
\begin{aligned}
d\left(Z_{u}\right)= & -\left(e^{-\int_{u}^{T} 2 \widehat{a}\left(\nu, S_{\nu}\right) \frac{d \nu}{\epsilon S_{\nu}}}\right) \frac{1}{2} e^{\int_{u}^{T} 2 \widehat{a}\left(\nu, S_{\nu}\right) \frac{d \nu}{\epsilon S_{\nu}}} \sigma^{2} S_{u}^{2+2 \gamma} d u \\
& +2 \widehat{a}\left(u, S_{u}\right) \frac{d u}{\epsilon S_{u}} e^{-\int_{u}^{T} 2 \widehat{a}\left(\nu, S_{\nu}\right) \frac{d \nu}{\epsilon S_{\nu}}}\left(\int_{u}^{T} \frac{1}{2} e^{\int_{\xi}^{T} 2 \widehat{a}\left(\nu, S_{\nu}\right) \frac{d \nu}{\epsilon S_{\nu}}} \sigma^{2} S_{\xi}^{2+2 \gamma} d \xi\right),
\end{aligned}
$$

so that

$$
d\left(Z_{u}\right)=-\frac{1}{2} \sigma^{2} S_{u}^{2+2 \gamma} d u+2 \widehat{a}\left(u, S_{u}\right) \frac{d u}{\epsilon S_{u}}\left(\int_{u}^{T} \frac{1}{2} e^{-\int_{u}^{\xi} 2 \widehat{a}\left(\nu, S_{\nu}\right) \frac{d \nu}{\epsilon S_{\nu}}} \sigma^{2} S_{\xi}^{2+2 \gamma} d \xi\right) .
$$

The coefficients of $d u$ are continuous. We calculate the (general) infinitesimal generator at $\widetilde{a}$ :

$$
\begin{aligned}
& \lim _{r \downarrow 0} \frac{E\left[\widetilde{a}\left(t+r, S_{t+r}\right) \mid S_{t}=S\right]-\widetilde{a}(t, S)}{r} \\
& =\lim _{r \downarrow 0} \frac{E\left[E\left[Z_{t+r} \mid S_{t+r}=S_{t+r}\right] \mid S_{t}=S\right]-E\left[Z_{t} \mid S_{t}=S\right]}{r} \\
& =\lim _{r \downarrow 0} \frac{\int_{t}^{t+r} E_{t, S}\left(-\frac{1}{2} \sigma^{2} S_{u}^{2+2 \gamma}+2 \widehat{a}\left(u, S_{u}\right) \frac{1}{\epsilon S_{u}} Z_{t}\right)}{r} \\
& =E_{t, S}\left(-\frac{1}{2} \sigma^{2} S_{t}^{2+2 \gamma}+2 \widehat{a}\left(t, S_{t}\right) \frac{1}{\epsilon S_{t}} Z_{t}\right) \\
& =-\frac{1}{2} \sigma^{2} S_{t}^{2+2 \gamma}+2 \widehat{a}\left(t, S_{t}\right) \frac{1}{\epsilon S_{t}} E_{t, S}\left(Z_{t}\right) .
\end{aligned}
$$

Since the above function is continuous, so is $L(\widehat{a})$. We already know $\widehat{a}$ is continuously differentiable in $t$ and $\frac{1}{2} \sigma^{2} S^{2+2 \gamma}$ is continuous and nonzero in the interior $(S>0)$. If $L(\widehat{a})(t, S)=\frac{\partial \widehat{a}}{\partial t}(t, S)+\frac{1}{2} \sigma^{2} S^{2+2 \gamma} \frac{\partial^{2} \widehat{a}}{\partial S^{2}}(t, S)$ with Sobolev derivatives, then $\widehat{a}$ is $C^{2}$ with respect to $S$ (for $S>0$ ). This follows from the fact that if a function has a continuous derivative in the Sobolev sense, then it is $C^{1}$. To prove this, one can use [9, 5.8.3 Theorem 5], and extend continuously the classical derivative $\left(f_{\text {Sob }}^{\prime} \in C \Rightarrow f_{\text {Sob }}^{\prime} \in L_{\text {loc }}^{\infty}\right.$ and $f$ is locally Lipschitz) to show it is defined everywhere.

Now, to complete the proof it is sufficient to show that $L(\widehat{a})(t, S)=$ $\frac{\partial \widehat{a}}{\partial t}(t, S)+\frac{1}{2} \sigma^{2} S^{2+2 \gamma} \frac{\partial^{2} \widehat{a}}{\partial S^{2}}(t, S)$ with Sobolev derivatives. Let $\bar{B}(x, r) \subset[0, \infty)$ be a closed ball with center $x$ and radius $r$. Let us define 


$$
\begin{aligned}
& \widehat{a}_{x, r}(t, S) \\
& \quad= \begin{cases}\widehat{a}(t, S), & (t, S) \in \bar{B}(x, r), \\
\widehat{a}\left(t+\frac{t-x_{1}}{|(t, S)-x|} r, x+\frac{t-x_{2}}{|(t, S)-x|} r\right) \frac{2 r-|(t, S)-x|}{r}, & (t, S) \in \bar{B}(x, 2 r) \backslash \bar{B}(x, r), \\
0 & (t, S) \notin \bar{B}(x, 2 r) .\end{cases}
\end{aligned}
$$

It is a continuous function with compact support $\bar{B}(x, 2 r)$. Using a standard mollifier family $\left(\phi_{1 / n}\right)_{n \in \mathbb{N}}$, we get the sequence $\left(\widehat{a}_{x, r} * \phi_{1 / n}\right)_{n \in \mathbb{N}} \subset C_{0}^{\infty}$ uniformly integrable and convergent to $\widehat{a}_{x, r}$. From Dynkin's formula,

$$
\begin{aligned}
\lim _{s \downarrow 0} \frac{E\left[\widehat{a}_{r, x} * \phi_{1 / n}\left(t+s, S_{t+s}\right) \mid S_{t}=S\right]-\widehat{a}_{x, r} * \phi_{1 / n}(t, S)}{s} \\
=L\left(\widehat{a}_{x, r} * \phi_{1 / n}\right)(t, S) \\
=\frac{\partial \widehat{a}_{x, r} * \phi_{1 / n}}{\partial t}(t, S)+\frac{1}{2} \sigma^{2} S^{2+2 \gamma} \frac{\partial^{2} \widehat{a}_{x, r} * \phi_{1 / n}}{\partial S^{2}}(t, S) .
\end{aligned}
$$

We can use (3.20) and take the $\operatorname{limit}_{n \rightarrow \infty}$ in $L\left(\widehat{a}_{x, r} * \phi_{1 / n}\right)(t, S)$ :

$$
\begin{aligned}
& \lim _{n \rightarrow \infty} \lim _{s \downarrow 0} \frac{E\left[\widehat{a}_{x, r} * \phi_{1 / n}\left(S_{t+s}, t+s\right) \mid S_{t}=S\right]-\widehat{a}_{x, r} * \phi_{1 / n}(S, t)}{s} \\
&=\lim _{n \rightarrow \infty} L\left(\widehat{a}_{x, r} * \phi_{1 / n}\right)(t, S) .
\end{aligned}
$$

Now $\left(\widehat{a}_{x, r} * \phi_{1 / n}\right)_{n \in \mathbb{N}}$ is uniformly convergent, integrable and we can interchange the limits. Because this sequence is also uniformly integrable, we can move the limit under the expectation sign:

$$
\begin{aligned}
L(\widehat{a})(t, S) & =\lim _{s \downarrow 0} \lim _{n \rightarrow \infty} \frac{E\left[\widehat{a}_{x, r} * \phi_{1 / n}\left(S_{t+s}, t+s\right) \mid S_{t}=S\right]-\widehat{a}_{x, r} * \phi_{1 / n}(S, t)}{s} \\
& =\lim _{n \rightarrow \infty} L\left(\widehat{a}_{x, r} * \phi_{1 / n}\right)(t, S) .
\end{aligned}
$$

As $\frac{1}{2} \sigma^{2} S^{2+2 \gamma}$ is continuous, and so is $\frac{\partial \widehat{a}_{x, r}}{\partial t}$, the following is also continuous:

$$
\frac{L\left(\widehat{a}_{x, r}\right)(t, S)-\frac{\partial \widehat{a}_{x, r}}{\partial t}}{\frac{1}{2} \sigma^{2} S^{2+2 \gamma}}=\lim _{n \rightarrow \infty} \frac{L\left(\widehat{a}_{x, r} * \phi_{1 / n}\right)(t, S)-\frac{\partial \widehat{a}_{x, r}}{\partial t} * \phi_{1 / n}}{\frac{1}{2} \sigma^{2} S^{2+2 \gamma}} .
$$

From 3.21 this is equal to $\lim _{n \rightarrow \infty} \frac{\partial^{2} \widehat{a}_{x, r} * \phi_{1 / n}}{\partial S^{2}}=\lim _{n \rightarrow \infty} \widehat{a}_{x, r} * \frac{\partial^{2} \phi_{1 / n}}{\partial S^{2}}$. Additionally, $\left(\widehat{a}_{x, r} * \phi_{1 / n}\right)_{n \in \mathbb{N}}$ is uniformly bounded by an integrable function $\max _{S \in \bar{B}(x, r)} \widehat{a}(t, S)$ on $\bar{B}(x, 2 r+1 / n)$ and 0 elsewhere. For every $g \in C_{0}^{\infty}$ we can use the dominated convergence theorem:

$$
\begin{aligned}
\int_{\mathbb{R}} g(S) \frac{L\left(\widehat{a}_{x, r}\right)(t, S)-\frac{\partial \widehat{a}_{x, r}}{\partial t}}{\frac{1}{2} \sigma^{2} S^{2+2 \gamma}} d S & =\int_{\mathbb{R}} \lim _{n \rightarrow \infty} \widehat{a}_{x, r} * \frac{\partial^{2} \phi_{1 / n}}{\partial S^{2}}(t, S) g(S) d S \\
& =\lim _{n \rightarrow \infty} \int_{\mathbb{R}} \widehat{a}_{x, r} * \frac{\partial^{2} \phi_{1 / n}}{\partial S^{2}}(t, S) g(S) d S .
\end{aligned}
$$


By integration by parts,

$$
\int_{\mathbb{R}} g(S) \frac{L\left(\widehat{a}_{x, r}\right)(t, S)-\frac{\partial \widehat{a}_{x, r}}{\partial t}}{\frac{1}{2} \sigma^{2} S^{2+2 \gamma}} d S=\lim _{n \rightarrow \infty} \int_{\mathbb{R}}\left(\widehat{a}_{x, r} * \phi_{1 / n}\right)(t, S) \frac{\partial^{2} g}{\partial S^{2}}(S) d S .
$$

We can use the dominated convergence theorem in another way:

$$
\begin{aligned}
\int_{\mathbb{R}} g(S) \frac{L\left(\widehat{a}_{x, r}\right)(t, S)-\frac{\partial \widehat{a}_{x, r}}{\partial t}}{\frac{1}{2} \sigma^{2} S^{2+2 \gamma}} d S & =\int_{\mathbb{R}} \lim _{n \rightarrow \infty}\left(\widehat{a}_{x, r} * \phi_{1 / n}\right)(t, S) \frac{\partial^{2} g}{\partial S^{2}}(S) d S \\
& =\int_{\mathbb{R}}\left(\widehat{a}_{x, r}\right)(t, S) \frac{\partial^{2} g}{\partial S^{2}}(S) d S .
\end{aligned}
$$

The function $\frac{L\left(\widehat{a}_{x, r}\right)(t, S)-\frac{\partial \widehat{a}_{x, r}}{\partial t}}{\frac{1}{2} \sigma^{2} S^{2+2 \gamma}}$ in the open ball $B(x, r)$ is a derivative in the Sobolev sense, directly from the definition. By definition $L\left(\widehat{a}_{x, r}\right)$ is equal to $L(\widehat{a})$ in $B(x, r)$, and similarly for $\frac{\partial \widehat{a}_{x, r}}{\partial t}$. Therefore, $\widehat{a}$ has a continuous second derivative in the Sobolev sense, thus, it is $C^{2}$ in that variable.

Lemma 3.9. If $\widehat{a}$ is $C^{2}$ and nonnegative, then

$$
\widetilde{a}(t, S)=\frac{1}{2} E_{t, S}\left(\int_{t}^{T} e^{-\int_{t}^{u} 2 \widehat{a}\left(\nu, S_{\nu}\right) \frac{d \nu}{\epsilon S_{\nu}}} \sigma^{2} S_{u}^{2+2 \gamma} d u+\frac{\epsilon S_{T}}{2 T^{\prime}} e^{-\int_{t}^{T} 2 \widehat{a}\left(\nu, S_{\nu}\right) \frac{d \nu}{\epsilon S_{\nu}}}\right)
$$

is $C^{2}$ and it solves the auxiliary equation (3.11).

Proposition 3.10. The PDE (3.1) with the terminal condition

$$
a(T, \cdot) \equiv 0
$$

has a unique solution.

Proof. Define

$$
\Psi(\alpha)(t, S)=\frac{1}{2} E_{t, S}\left(\int_{t}^{T} e^{-\int_{t}^{u} 2 \alpha\left(\nu, S_{\nu}\right) \frac{d \nu}{\epsilon S_{\nu}}} \sigma^{2} S_{u}^{2+2 \gamma} d u\right)
$$

where $\alpha$ satisfies the terminal condition $\alpha(T, \cdot) \equiv 0$. Lemma 3.8 proves that $\Psi(\widehat{a}(\cdot, \cdot))$ is a well defined solution of the auxiliary PDE (3.10). The terminal condition $\Psi(\alpha)(T, S)=0$ is also fulfilled. Then from Lemma 3.3 it can be deduced that a fixed point of $\Psi$ is a solution of (3.1). Conversely, a solution of (3.1) must be a fixed point of $\Psi$. It is clear that $\Psi(\cdot) \geq 0$. We define recursively

$$
a^{(0)} \equiv 0, \quad a^{(n+1)}=\Psi\left(a^{(n)}\right) .
$$

We notice that $\Psi$ is nonincreasing:

$$
x \geq y \Rightarrow \Psi(x) \leq \Psi(y) .
$$

Since the arguments of $\Psi$ are functions, the inequality on the left side must hold for all arguments of $x, y$. We point out that if the left inequality is strict 
on some set of nonzero Lebesgue measure, the right inequality is strict. By using this inequality repeatedly, we get

$$
a^{(0)}=0 \leq a^{(2)} \leq a^{(1)}, \quad a^{(1)} \geq a^{(3)} \geq a^{(2)} .
$$

By induction using 3.23 one can prove

$$
a^{(2 n)} \leq a^{(2 n+2)} \leq a^{(2 n+1)}, \quad a^{(2 n)} \leq a^{(2 n+1)} \leq a^{(2 n-1)} .
$$

Since $a^{(2 n+1)}$ is decreasing and bounded from below by 0 , it has a limit $\bar{a}$ (in $\left.L^{1}\right)$. Similarly $a^{(2 n)}$ is increasing and bounded by any term of $a^{(2 n+1)}$, thus by $\bar{a}$. Therefore, $a^{(2 n)}$ has a limit $\underline{a}$ and $\underline{a} \leq \bar{a}$. By the monotone convergence theorem we have $\Psi(\bar{a})=\underline{a}$ and $\bar{a}=\Psi(\underline{a})$.

According to Lemma 3.8 ,

$$
\begin{aligned}
& 0=L(\bar{a})+\frac{1}{2} \sigma^{2} S^{2+2 \gamma}+\frac{4 \bar{a} \underline{a}}{S \epsilon}, \\
& 0=L(\underline{a})+\frac{1}{2} \sigma^{2} S^{2+2 \gamma}+\frac{4 \bar{a} \underline{a}}{S \epsilon} .
\end{aligned}
$$

Subtracting yields

$$
0=L(\bar{a}-\underline{a}) \Leftrightarrow \forall_{t} E_{t, S}\left(\bar{a}\left(S_{t}, t\right)-\underline{a}\left(S_{t}, t\right)\right) \equiv \text { const. }
$$

We recall the terminal condition $a(S, T)=0$ (satisfied by every $\Psi(\alpha)$ ) and the inequality $\bar{a}-\underline{a} \geq 0$. By using these and the above equation, we obtain $\bar{a}=\underline{a}$. Therefore, we have at least one solution.

Intuitively there should be only one solution for a hedging problem. This turns out to be true, as will be proved shortly. In fact we will prove that the choice of $a_{0} \geq 0$ is irrelevant, which is important from the numerical perspective. We suppose there is a certain solution $\tilde{a}$ of PDE and a fixed point of $\Psi$. Because $\tilde{a}=\Psi(\tilde{a})$, also $\tilde{a} \geq 0$. We recall that $\Psi$ is nonincreasing, therefore

$$
a^{(0)}=0 \geq \tilde{a}=\Psi(\tilde{a}) \geq \Psi(0)=a^{(1)} .
$$

Again by induction on $n$,

$$
a^{(2 n)} \geq \tilde{a} \geq a^{(2 n+1)} .
$$

Letting $n \rightarrow \infty$ and applying the monotone convergence theorem yields $\bar{a}=\tilde{a}$.

The following lemma can be proved using the same technique.

Lemma 3.11. The PDE (3.6) with the terminal condition

$$
\alpha(T, \cdot) \equiv \frac{\epsilon S_{T}}{2 T^{\prime}}
$$

has a unique solution.

We can use an analogue of Feynman-Kac representation for $b$ and $c$ as we did for $a$ for the auxiliary PDE, due to condition (1.7) on $\theta$. There are 
no essential differences in the proofs, so we omit them. The results are as follows:

LEMma 3.12. The unique solution of equation (3.2) is given by

$$
b(t, S)=-E_{t, S}\left(\int_{t}^{T} e^{-\int_{t}^{u} 2 a\left(\nu, S_{\nu}\right) \frac{d \nu}{\epsilon S_{\nu}}} \theta\left(u, S_{u}\right) \sigma^{2} S_{u}^{2+2 \gamma} d u\right) .
$$

The unique solution of equation (3.3) is given by

$$
c(t, S)=\frac{1}{2} E_{t, S}\left(\int_{t}^{T}\left(\theta\left(u, S_{u}\right)^{2} \sigma^{2} S_{u}^{2+2 \gamma}-\frac{2 b\left(u, S_{u}\right)^{2}}{\epsilon S_{u}}\right) d u\right) .
$$

Notice that the above equations are valid for the case with the liquidity cost of selling a final portfolio if we substitute $\bar{a}$ in place of $a$.

\subsection{Solution of HJB is the true value function}

Proposition 3.13. The function $V$ defined in (3.5) with $a=\lim _{n \rightarrow \infty} a_{n}$ given by Proposition 3.10 and $b, c$ given by (3.25) and (3.26) is the true value function of the stochastic control problem 2.3).

Proof. Since $a, b, c$ are $C^{2}$ (as shown in Lemma 3.8), so is $V=H^{2} a+$ $H b+c$ (in the $H$ variable too). We have unique solutions a (Proposition 3.10 and $b, c$ (Lemma 3.12) of (3.1), (3.2), (3.3) respectively, thus $V$ solves (2.11) by Lemma 3.1 . Using Lemma 2.3 we find that it also solves the HamiltonJacobi-Bellman equation (2.5) with $h$ where the minimum is attained.

Recall the equation 2.3) for $V(H, S, t)$ :

$$
\inf _{h \in \mathcal{H}} \frac{1}{2} E_{t, S, H}\left(\int_{t}^{T}\left(\theta\left(u, S_{u}\right)-H_{u}\right)^{2} \sigma^{2} S^{2+2 \gamma} d u+\int_{t}^{T} S_{u} l\left(h_{u}\right) d u\right) .
$$

Clearly, for any strategy $h \in \mathcal{H}$,

$$
\begin{aligned}
& V\left(H_{t}, S_{t}, t\right) \\
& \quad \leq \frac{1}{2} E_{t, S, H}\left(\int_{t}^{T}\left(\theta\left(u, S_{u}\right)-H_{u}\right)^{2} \sigma^{2} S_{u}^{2+2 \gamma} d u+\int_{t}^{T} S_{u} l\left(h_{u}\right) d u \mid \mathcal{F}_{t}\right) \\
& \quad \leq \frac{1}{2} E_{t, S, H}\left(\int_{0}^{T} 2\left(\theta\left(u, S_{u}\right)^{2}+H_{u}^{2}\right) \sigma^{2} S_{u}^{2+2 \gamma} d u+\int_{0}^{T} S_{u} l\left(h_{u}\right) d u \mid \mathcal{F}_{t}\right) .
\end{aligned}
$$

Now, we use (1.4) (the condition for being in $\mathcal{H}$ ):

$$
\int_{0}^{T} H_{t}^{2} S_{t}^{2} d S_{t}<\infty, \quad \int_{0}^{T} l\left(h_{t}\right) S_{t} d t<\infty,
$$

and the condition 1.7$)$ on $\theta$ :

$$
\exists_{C, \alpha>0} \forall_{S>0, t \in[0, T]}: \quad|\theta(t, S)|<C\left(1+S^{\alpha}\right) .
$$


Summing up, the process $V\left(H_{t}, S_{t}, t\right)$ is bounded by a uniformly integrable martingale. In fact the condition for being in $\mathcal{H}$ was needed only to ensure that these integrals exist and are finite. Strategies with infinite integrals will not achieve the infimum, since e.g. a constant strategy has finite integrals. Actually we find the infimum over a larger set. According to Ito's lemma the following process is a local martingale plus a nondecreasing term (DoobMeyer decomposition):

$$
Y_{t}=\frac{1}{2} E_{t, S, H}\left(\int_{0}^{t}\left(\theta\left(u, S_{u}\right)-H_{u}\right)^{2} \sigma^{2} S_{u}^{2+2 \gamma} d u+\int_{0}^{t} S_{u} l\left(h_{u}\right) d u\right)+V\left(t, H_{t}, S_{t}\right) .
$$

This process is connected with a sequence of stopping times $\tau_{m}$ approaching $T$, which reduces the stopped local martingale to a martingale. Due to the nondecreasing term we have the inequality between $Y_{t}$ stopped by $\tau_{m}$ at 0 and the one stopped at $T\left(\tau_{m} \wedge T=\tau_{M}\right.$ because $\tau_{m} \leq T$, and $\tau_{m} \wedge 0=0$ because $\left.\tau_{m} \geq 0\right)$ :

$$
\begin{aligned}
V\left(0, H_{0}, S_{0}\right) \leq & \frac{1}{2} E_{t, S, H}\left(\int_{0}^{\tau_{m}}\left(\theta\left(u, S_{u}\right)-H_{u}\right)^{2} \sigma^{2} S_{u}^{2+2 \gamma} d u+\int_{0}^{\tau_{m}} S_{u} l\left(h_{u}\right) d u\right) \\
& +V\left(\tau_{m}, H_{\tau_{m}}, S_{\tau_{m}}\right) .
\end{aligned}
$$

Note that $V\left(T, H_{T}, S_{T}\right)=0$ by definition. As $\tau_{m} \rightarrow T$, the integrals monotonically converge (in $L^{1}$ ):

$$
V\left(0, H_{0}, S_{0}\right) \leq \frac{1}{2} E_{t, S, H}\left(\int_{0}^{T}\left(\theta\left(u, S_{u}\right)-H_{u}\right)^{2} \sigma^{2} S_{u}^{2+2 \gamma} d u+\int_{0}^{T} S_{u} l\left(h_{u}\right) d u\right) .
$$

Therefore, the assumptions of Lemma 2.1 are fulfilled, and $V$ must be the true value function.

Proposition 3.14. The function $V$ defined in (3.9) with $\bar{a}=\lim _{n \rightarrow \infty} a_{n}$ given by Proposition 3.11, with

$$
\begin{aligned}
a_{n+1} & =\Psi\left(a_{n}\right) \\
& =\frac{1}{2} E_{t, S, H}\left(\int_{t}^{T} e^{-\int_{t}^{u} 2 a_{n}\left(\nu, S_{\nu}\right) \frac{d \nu}{\epsilon S_{\nu}}} \sigma^{2} S_{u}^{2+2 \gamma} d u+\frac{\epsilon S_{T}}{2 T^{\prime}} e^{-\int_{t}^{T} 2 a_{n}\left(\nu, S_{\nu}\right) \frac{d \nu}{\epsilon S_{\nu}}}\right)
\end{aligned}
$$

and $\bar{b}, \bar{c}$ given by (3.25) and (3.26) with $\bar{a}$ instead of $a$,

$$
\begin{aligned}
& \bar{b}(t, S)=-E_{t, S, H}\left(\int_{t}^{T} e^{-\int_{t}^{u} 2 \bar{a}\left(\nu, S_{\nu}\right) \frac{d \nu}{\epsilon S_{\nu}}} \theta\left(u, S_{u}\right) \sigma^{2} S_{u}^{2+2 \gamma} d u\right), \\
& \bar{c}(t, S)=\frac{1}{2} E_{t, S, H}\left(\int_{t}^{T}\left(\theta^{2}\left(u, S_{u}\right) \sigma^{2} S_{u}^{2+2 \gamma}-\frac{2 \bar{b}^{2}\left(u, S_{u}\right)}{\epsilon S_{u}}\right) d u\right)
\end{aligned}
$$

is the true value function of the stochastic control problem (2.4). 
4. Numerical results. In this section we discuss a numerical solution to the problem and present some results. Our test problem is the hedging of a standard European call option with strike price 1. The numerical results we obtain are consistent with the presented theory, e.g. the inequalities between subsequences proven in Proposition 3.10 .

The computations follow the lines of the theoretical proofs. We solve the original PDE (2.11) by using (3.5) and then solving (3.1)-(3.3). To solve (3.1) we use the function $\Psi$ used in Proposition 3.10 (defined in (3.22)). However, we do not calculate stochastic integrals in the main procedure. The Crank-Nicolson scheme is used for the equivalent PDE (3.10), because it is much faster and provides the same results (Lemma 3.8), at least for small numbers of iterations ( $n$ in Proposition 3.10). To solve the algebraic problem generated by the Crank-Nicolson scheme we use a (Thomas) tridiagonal matrix algorithm. The boundary conditions are as follows:

$$
\begin{aligned}
\forall_{0 \leq t \leq T}: & a(0, t)=0, \\
\forall_{0 \leq S \leq S_{\max }:} & a(S, T)=0, \\
\forall_{0 \leq t \leq T}: & a\left(S_{\max +}, t\right)=a\left(S_{\max }, t\right)+\left(a\left(S_{\max }, t\right)-a\left(S_{\max -}, t\right)\right),
\end{aligned}
$$

where $S_{\max }$ - is the second largest $S$ value in the mesh, $S_{\max }$ is the maximal value for $S$ in the mesh, and $S_{\max }+$ is just $S_{\max }$ plus one step $\Delta S . S_{\max +}$ is important, because knowing $a\left(S_{\max +}, t\right)$ is required to calculate $\frac{\partial^{2} a}{\partial S^{2}}\left(S_{\max }, t\right)$. The last condition was added, because the theory does not provide any obvious prospects for the value $a\left(S_{\max +}, t\right)$.

To check if the Crank-Nicolson method works well in our setting we calculate and compare the first 10 iterations with stochastic integrals with good results (low MSE). Since the comparison of solving methods for SDE/PDE problems is not the main topic of this paper, we do not report the results.

In this section the best hedge is considered to be a possibly fractional number of shares for which the value function is minimal $\left(\frac{\partial V}{\partial H}=0\right.$, due to (3.5)). Equivalently it starts with $H_{t}=H$ for which the optimal strategy does not buy or sell shares at the start $\left(h_{t}=0\right)$. We will also call it the target portfolio. Combining (2.10) and (3.5) yields

$$
2 a(S, t) H_{t}^{*}+b(S, t)=0, \quad H_{t}^{*}=-\frac{b(S, t)}{2 a(S, t)} .
$$

We calculate the optimal hedging strategy for the European call option for $K=1,0<S_{0}<100$. We choose $\epsilon=6 \%$ as in [21]. We use the method of calculating the price or hedge (in a liquid environment) of options in the CEV model from [19].

The accurate estimation of $\epsilon$ and other similar parameters can be found in [4]. We point out that the value of $\epsilon$ is probably linked to the current market situation (daily volume of trade, RoC etc.) to some extent. 


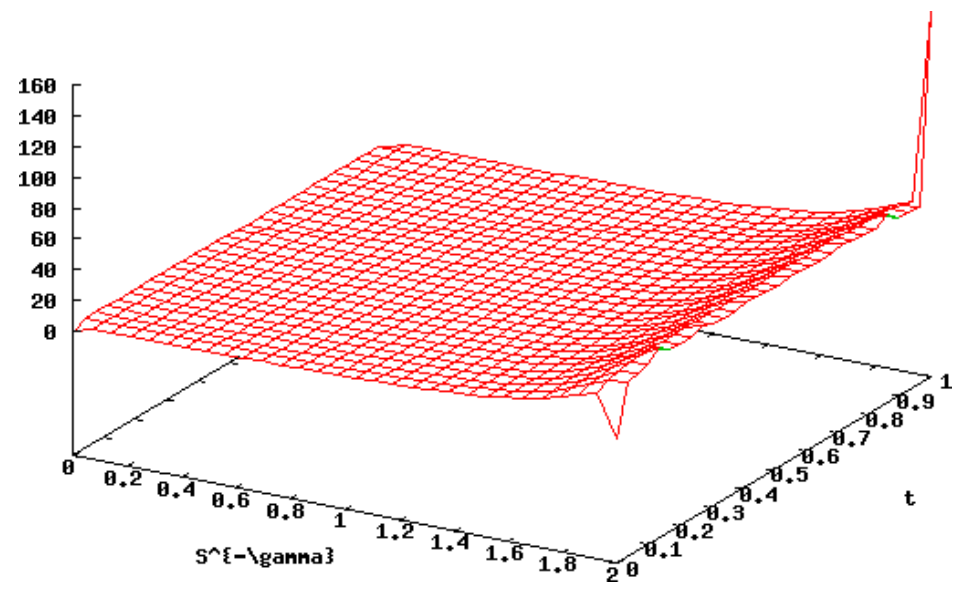

Fig. 1. The function $a$ approximated by the scheme described in Section 4

Approximation of $a$ (Figure 1) yields a smooth surface. Only 20 iterations of the previously described iterative algorithm were needed to achieve acceptable accuracy. For a greater number $n$ of iterations, (3.24) will no longer hold, as the gap between $a^{2 n}$ and $a^{2 n+1}$ is small compared to errors induced by discretization and rounding errors.

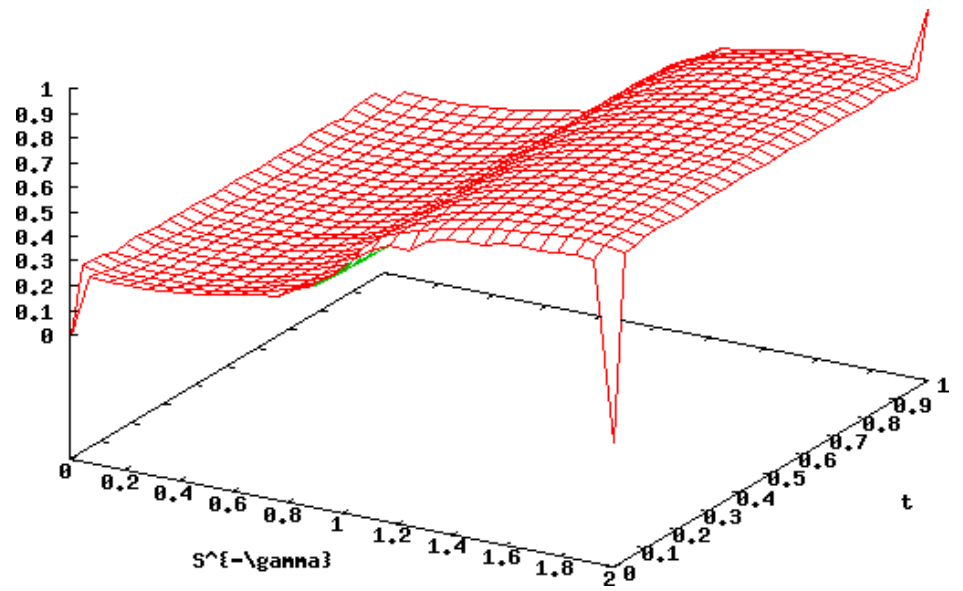

Fig. 2. The current hedge $\left(H_{t}\right)$ such that $h_{t}=0$, i.e. with no need to rebalance the hedging portfolio

Figure 2 presents the values of the most desirable hedging portfolio (in fraction of underlying $a$ per option) at time $t$ and $S_{t}=S$. By the most desirable hedging portfolio we mean a hedging portfolio which need not be rebalanced immediately $\left(h_{t}=0\right)$.

In Figure 3 we present the differences between the most desirable hedging portfolio and the classical hedge $\theta$ under $\mathrm{CEV}$. As one may expect, the largest 


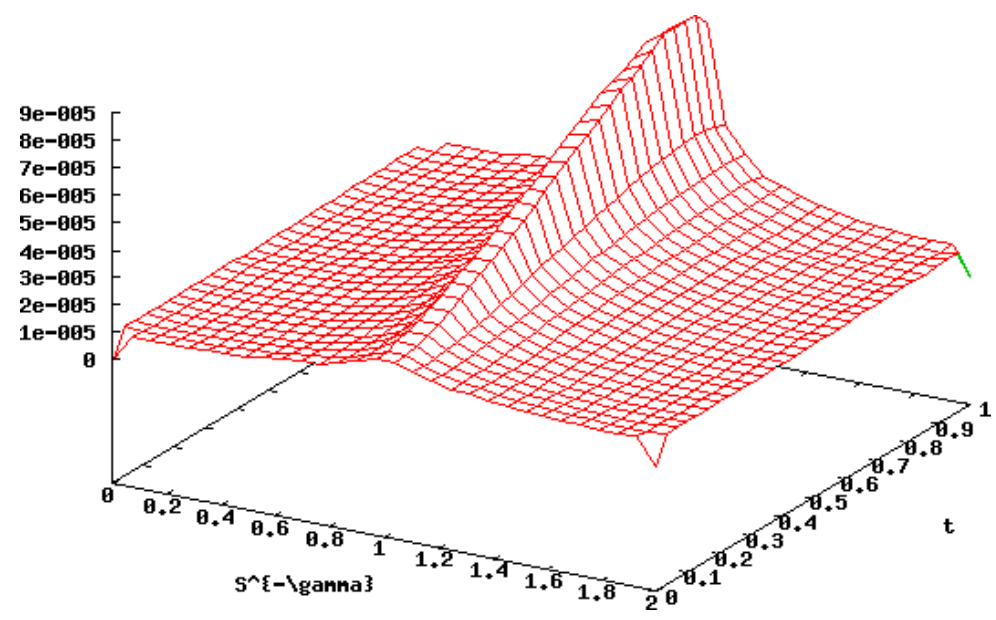

Fig. 3. The difference between our hedge $\left(H_{t}\right)$ such that $h_{t}=0$ and the classical hedge $(\theta)$

values are close to the option's strike price $K=1$. Even a small change in the asset price close to the strike price forces the investor to rebalance his portfolio. Therefore, our framework will be most useful for an asset price close to the strike price. Away from the strike price, adjustments are not significant.

5. Bounds of the function $V$. Recall the definition $(2.3)$ of the value function $V$ :

$$
\inf _{h \in \mathcal{H}} \frac{1}{2} E_{t, S, H}\left(\int_{t}^{T}\left(\theta\left(u, S_{u}\right)-H_{u}\right)^{2} \sigma^{2} S_{u}^{2+2 \gamma} d u+\int_{t}^{T} S_{u} l\left(h_{u}\right) d u\right) .
$$

Since all terms are nonnegative, $V$ is bounded from below by 0 . The infimum over all strategies $h$ is no greater than the value for any particular $h$, so the value of the above equation with a chosen $h$ is an upper bound of $V$. Now we could write down this optimal $h$ and calculate this bound, but we want to show some intuitive reasons for making this particular choice.

5.1. Intuition behind the choice of $\bar{h}$. The HJB equation for our problem is (see 2.5 )

$$
0=\inf _{h}\left(h V_{H}+L(V)+\frac{1}{2} \sigma^{2} S^{2+2 \gamma}(\theta(t, S)-H)^{2}+S l(h)\right) .
$$

The term $L(V)$ describes the change of the expected value of $V$ in time. In an ideal case this term is 0 or at least very small. We will assume $L(V)=0$ for a moment. Moreover, we substitute $l(\bar{h})=\epsilon \bar{h}^{2} / 2$ into the equation:

$$
0=\inf _{\tilde{h}}\left(\tilde{h} V_{H}+\frac{1}{2} \sigma^{2} S^{2+2 \gamma}(\theta(t, S)-H)^{2}+\frac{S \epsilon \tilde{h}^{2}}{2}\right) .
$$


We find $\bar{h}$ in the same way as we dealt with $h$ previously:

$$
\bar{h}=\underset{\tilde{h}}{\arg \min }\left(\tilde{h} V_{H}+L(V)+\frac{1}{2} \sigma^{2} S^{2+2 \gamma}(\theta(t, S)-H)^{2}+\frac{S \epsilon \tilde{h}^{2}}{2}\right) .
$$

Quadratic functions are convex, so they have one extremum, and it is a minimum in this case. We calculate the derivative of the term inside the argmin with respect to $\tilde{h}$ :

$$
0=V_{H}+S \epsilon \bar{h}, \quad \bar{h}=-\frac{V_{H}}{S \epsilon} .
$$

In this form, $\bar{h}$ still has a term $V_{H}$ inside. If we use this form, we will obtain a term $V_{H}^{2}$ in a PDE later. To avoid problems we encountered earlier, we will use the HJB equation (in the form assumed in this section, not a true one) again. After substituting $\bar{h}$, it looks as follows:

$$
\begin{aligned}
& 0=-\frac{V_{H}}{S \epsilon} V_{H}+\frac{1}{2} \sigma^{2} S^{2+2 \gamma}(\theta(t, S)-H)^{2}+\frac{S \epsilon\left(-\frac{V_{H}}{S \epsilon}\right)^{2}}{2}, \\
& \frac{\bar{h}^{2} S \epsilon}{2}=\frac{V_{H}^{2}}{2 S \epsilon}=\frac{1}{2} \sigma^{2} S^{2+2 \gamma}(\theta(t, S)-H)^{2} \\
& \bar{h}^{2}=\frac{\sigma^{2} S^{2+2 \gamma}(\theta(t, S)-H)^{2}}{S \epsilon} \\
& \bar{h}=\sigma S^{1 / 2+\gamma}(\theta(t, S)-H) \epsilon^{-1 / 2} .
\end{aligned}
$$

This $\bar{h}$ will be used to calculate the upper bound.

5.2. $V$ is $o\left(\epsilon^{k}\right)$

LEMMA 5.1. The value function $V$ is bounded from above by a finite integral:

$$
V(H, S, t) \leq E_{t, S}\left(q\left(T, S_{T}\right)-q(t, S)-\left(S_{T}-S\right) \theta(t, S)\right)<\infty .
$$

Proof. We calculate the value $V$ of a strategy $\bar{h}$ by substituting into the original equation (2.1):

$$
v(H, S, t \mid \bar{h})=E_{t, S, H}\left(\int_{t}^{T} \sigma^{2} S_{\nu}^{2+2 \gamma}\left(\theta\left(\nu, S_{\nu}\right)-H_{\nu}\right)^{2} d \nu\right) .
$$

Recall

$$
d H_{\nu}=\bar{h}_{\nu} d \nu=\sigma S^{1 / 2+\gamma}\left(\theta\left(\nu, S_{\nu}\right)-H_{\nu}\right) \epsilon^{-1 / 2} d \nu .
$$

The main problem in determining the asymptotic behaviour of $V$ (as a function of $\epsilon$ ) lies in $H_{\nu}$, as it contains $\epsilon$ in hidden form. In order to get rid of this problematic term we differentiate the integrand, then build and solve the resulting SDE and finish the calculations. Let us define

$$
Y_{\nu}=\theta\left(\nu, S_{\nu}\right)-H_{\nu}
$$


$S_{\nu}, H_{\nu}$ still satisfy the initial conditions $H_{t}=H, S_{t}=S$. Before we start calculating $d Y_{t}$, it is important to calculate the derivative of $\theta\left(\nu, S_{\nu}\right)=\frac{\partial q_{\nu}}{\partial S}$ :

$$
L(\theta)=L\left(\frac{\partial q_{\nu}}{\partial S}\right)=\frac{\partial L\left(q_{\nu}\right)}{\partial S}=\frac{\partial 0}{\partial S}=0 .
$$

This implies ( $L$ is the infinitesimal generator of $S_{\nu}$ ) that

$$
d \theta\left(\nu, S_{\nu}\right)=\sigma S_{\nu}^{1+\gamma} \frac{\partial \theta}{\partial S}\left(\nu, S_{\nu}\right) d W_{\nu}
$$

The stochastic derivative of $Y_{t}$ is given by

$$
d Y_{\nu}=\sigma S_{\nu}^{1+\gamma} \frac{\partial \theta\left(\nu, S_{\nu}\right)}{\partial S} d W_{\nu}-\sigma S_{\nu}^{1 / 2+\gamma}\left(\theta\left(\nu, S_{\nu}\right)-H_{\nu}\right) \epsilon^{-1 / 2} d \nu
$$

Now we write this in terms of $Y_{t}$ :

$$
d Y_{\nu}=\sigma S_{\nu}^{1+\gamma} \frac{\partial \theta\left(\nu, S_{\nu}\right)}{\partial S} d W_{\nu}-\sigma S_{\nu}^{1 / 2+\gamma} \epsilon^{-1 / 2} Y_{\nu} d \nu
$$

This (inhomogeneous) stochastic linear differential equation can be solved (uniquely) in a standard way (method of variation of parameters; for more details see e.g. [20, 5.1]):

$$
\begin{aligned}
Y_{\xi}= & C(H, S, t) \exp \left(-\sigma \epsilon^{-1 / 2} \int_{t}^{\xi} S_{\nu}^{1 / 2+\gamma} d \nu\right) \\
& +\sigma \int_{t}^{\xi} S_{\nu}^{1+\gamma} \frac{\partial \theta\left(\nu, S_{\nu}\right)}{\partial S} \exp \left(-\sigma \epsilon^{-1 / 2} \int_{\nu}^{\xi} S_{\mu}^{1 / 2+\gamma} d \mu\right) d W_{\nu} \\
& -\frac{1}{2} \sigma^{2} \int_{t}^{\xi} S_{\nu}^{2+2 \gamma}\left(\frac{\partial \theta\left(\nu, S_{\nu}\right)}{\partial S}\right)^{2} \exp \left(-\sigma \epsilon^{-1 / 2} \int_{\nu}^{\xi} S_{\mu}^{1 / 2+\gamma} d \mu\right) d \nu
\end{aligned}
$$

where $C(H, S, t)$ is given by the initial condition. In our problem,

$$
C(H, S, t)=\sigma S^{1+\gamma}(\theta(t, S)-H) .
$$

We rewrite equation (5.1) in terms of $Y_{t}$. This equation does not contain $H_{\nu}$, in fact the expected value need not be conditioned on $H_{t}=H$ at this point at all. We have

$$
\begin{aligned}
v(H, S, t \mid \bar{h}) & =E_{t, S, H}\left(\int_{t}^{T} \sigma^{2} S_{\xi}^{2+2 \gamma} Y_{\nu}^{2} d \nu\right) \\
= & E_{t, S, H}\left(\int _ { t } ^ { T } \sigma ^ { 2 } S _ { \xi } ^ { 2 + 2 \gamma } \left(C(H, S, t) \exp \left(-\sigma \epsilon^{-1 / 2} \int_{t}^{\xi} S_{\nu}^{1 / 2+\gamma} d \nu\right)\right.\right. \\
& \left.\left.+\sigma \int_{t}^{\xi} S_{\nu}^{1+\gamma} \frac{\partial \theta\left(\nu, S_{\nu}\right)}{\partial S} \exp \left(-\sigma \epsilon^{-1 / 2} \int_{\nu}^{\xi} S_{\mu}^{1 / 2+\gamma} d \mu\right) d W_{\nu}\right)^{2} d \xi\right) .
\end{aligned}
$$


We use the simple inequality $(a+b)^{2} \leq(a+b)^{2}+(a-b)^{2}=2 a^{2}+2 b^{2}$ :

$$
\begin{aligned}
& v(H, S, t \mid \bar{h}) \\
& \leq 2 E_{t, S, H}\left(\int_{t}^{T} \sigma^{2} S_{\xi}^{2+2 \gamma} C(H, S, t)^{2}\left(\exp \left(-\sigma \epsilon^{-1 / 2} \int_{t}^{\xi} S_{\nu}^{1 / 2+\gamma} d \nu\right)\right)^{2} d \xi\right) \\
& +2 E_{t, S, H}\left(\int _ { t } ^ { T } \sigma ^ { 2 } S _ { \xi } ^ { 2 + 2 \gamma } \left(\sigma \int_{t}^{\xi} S_{\nu}^{1+\gamma} \frac{\partial \theta\left(\nu, S_{\nu}\right)}{\partial S}\right.\right. \\
& \left.\left.\times \exp \left(-\sigma \epsilon^{-1 / 2} \int_{\nu}^{\xi} S_{\mu}^{1 / 2+\gamma} d \mu\right) d W_{\nu}\right)^{2} d \xi\right) .
\end{aligned}
$$

The first term is an integral in the time variable and its integrand is nonnegative, thus we can neglect the exponential term with a negative exponent, because it is smaller than 1 :

$$
E_{t, S, H}\left(\int_{t}^{T} \sigma^{2} S_{\xi}^{2+2 \gamma} C(H, S, t)^{2}\left(\exp \left(-\sigma \epsilon^{-1 / 2} \int_{t}^{\xi} S_{\nu}^{1 / 2+\gamma} d \nu\right)\right)^{2} d \xi\right)
$$

$$
\begin{aligned}
& \leq E_{t, S, H}\left(\int_{t}^{T} \sigma^{2} S_{\xi}^{2+2 \gamma} C(H, S, t)^{2} d \xi\right)=E_{t, S, H}\left(\left(\int_{t}^{T} \sigma S_{\xi}^{1+\gamma} C(H, S, t) d W_{\xi}\right)^{2}\right) \\
& =E_{t, S, H}\left(\left(\int_{t}^{T} C(H, S, t) d S_{\xi}\right)^{2}\right)=E_{t, S, H}\left(C(H, S, t)^{2}\left(S_{T}-S_{t}\right)^{2}\right)<\infty .
\end{aligned}
$$

In the calculation above we have used Ito's isometry as well. Ito's isometry assumptions are fulfilled since CEV's distribution higher moments are moments of a noncentral chi square [6] distribution (with appropriate parameters), which are finite (as in the proof of Lemma 3.8).

The second term of the right side of (5.3) can be transformed by Ito's isometry again, but in a different way:

$$
\begin{aligned}
& E_{t, S, H}\left(\int _ { t } ^ { T } \sigma ^ { 2 } S _ { \xi } ^ { 2 + 2 \gamma } \left(\sigma \int_{t}^{\xi} S_{\nu}^{1+\gamma} \frac{\partial \theta\left(\nu, S_{\nu}\right)}{\partial S}\right.\right.\left.\left.\times \exp \left(-\sigma \epsilon^{-1 / 2} \int_{\nu}^{\xi} S_{\mu}^{1 / 2+\gamma} d \mu\right) d W_{\nu}\right)^{2} d \xi\right) \\
&=E_{t, S, H}\left(\int _ { t } ^ { T } \left(\int_{t}^{\xi} \sigma^{2} S_{\xi}^{1+1 \gamma} S_{\nu}^{1+\gamma} \frac{\partial \theta\left(\nu, S_{\nu}\right)}{\partial S}\right.\right. \\
&\left.\left.\times \exp \left(-\sigma \epsilon^{-1 / 2} \int_{\nu}^{\xi} S_{\mu}^{1 / 2+\gamma} d \mu\right) d W_{\nu}\right)^{2} d \xi\right)=: I .
\end{aligned}
$$

We now move the expectation under the $d \xi$ integral (Tonelli's theorem 1, Th. 5.2.1] for $\sigma$-finite measures), then apply Ito's isometry to the inner $d S_{\nu}$ 
integral. Both theorems require an $L^{2}$ integrand. This can be achieved by combining the method used previously for the first term in (5.4), and the assumed condition on $\frac{\partial \theta\left(\nu, S_{\nu}\right)}{\partial S}$ in 1.8 . Thus

$$
\begin{aligned}
I=E_{t, S, H}\left(\int_{t}^{T} \int_{t}^{\xi} \sigma^{4} S_{\xi}^{2+2 \gamma} S_{\nu}^{2+2 \gamma}\right. & \left(\frac{\partial \theta\left(\nu, S_{\nu}\right)}{\partial S}\right)^{2} \\
& \left.\times \exp \left(-2 \sigma \epsilon^{-1 / 2} \int_{\nu}^{\xi} S_{\mu}^{1 / 2+\gamma} d \mu\right) d \nu d \xi\right) .
\end{aligned}
$$

We use the same trick with the exponential term as in (5.4) and again we move the expectation under the integral sign, use Ito's isometry, then move the expectation and use Ito's isometry once again:

$$
\begin{aligned}
I & \leq E_{t, S, H}\left(\int_{t}^{T} \sigma^{2} S_{\xi}^{2+2 \gamma} \sigma \int_{t}^{\xi} S_{\nu}^{2+2 \gamma}\left(\frac{\partial \theta\left(\nu, S_{\nu}\right)}{\partial S}\right)^{2} d \nu d \xi\right) \\
& =E_{t, S, H}\left(\left(\int_{t}^{T} \int_{t}^{\xi} \frac{\partial \theta\left(\nu, S_{\nu}\right)}{\partial S} d S_{\nu} d S_{\xi}\right)^{2}\right) .
\end{aligned}
$$

For a similar usage of (iterated) Ito's isometry plus theoretical justification see [8, Sect. 1.1]. Finally,

$$
\begin{aligned}
E_{t, S, H}\left(\left(\int_{t}^{T} \int_{t}^{\xi} \frac{\partial \theta\left(\nu, S_{\nu}\right)}{\partial S}\right.\right. & \left.\left.d S_{\nu} d S_{\xi}\right)^{2}\right)=E_{t, S, H}\left(\left(\int_{t}^{T}\left(\theta\left(\xi, S_{\xi}\right)-\theta\left(t, S_{t}\right)\right) d S_{\xi}\right)^{2}\right) \\
= & E_{t, S, H}\left(\left(\int_{t}^{T}\left(\theta\left(\xi, S_{\xi}\right)-\theta\left(t, S_{t}\right)\right) d S_{\xi}\right)^{2}\right) \\
& =E_{t, S, H}\left(q\left(T, S_{T}\right)-q(t, S)-\left(S_{T}-S\right) \theta(t, S)\right)<\infty .
\end{aligned}
$$

Because CEV higher noncentral moments are moments of a noncentral chi square distribution [6] (with appropriate parameters), and the latter have finite noncentral moments, the right side of the equation is finite. The formal proof is almost identical to the one of Lemma 3.8.

Proposition 5.2. The value function $V$ of problem (2.1) is o $\left.\epsilon^{k}\right)$.

Proof. Recall the estimate for $v(H, S, t \mid \bar{h}) \geq V(H, S, t)$ from Lemma 5.1. given in (5.3). Both terms on the right hand side of that equation are of the type $\exp \left(-D \epsilon^{-1 / 2}\right)$, where $D$ is a nonnegative, $\epsilon$-independent function. As $\epsilon \downarrow 0$, we have $-D \epsilon^{-1 / 2} \downarrow-\infty$, thus $\exp \left(-D \epsilon^{-1 / 2}\right) \downarrow 0$. In the previous subsection we proved that the integrals of the expressions above are finite, thus, due to Lebesgue's monotone convergence theorem, $v(H, S, t \mid \bar{h}) \downarrow 0$. Since $v(H, S, t \mid \bar{h}) \geq V(H, S, t) \geq 0$, also $V(H, S, t) \downarrow 0$. 
Now, we show that $(H, S, \mid \bar{h}) \in o\left(\epsilon^{k / 2}\right)$ for all positive integers $k$. We use de l'Hospital rule:

$$
\begin{aligned}
\lim _{\epsilon \downarrow 0} \frac{\exp \left(-D \epsilon^{-1 / 2}\right)}{\epsilon^{k / 2}} & =\lim _{\epsilon \downarrow 0} \frac{\epsilon^{-k / 2}}{\exp \left(D \epsilon^{-1 / 2}\right)} \\
& =\lim _{\epsilon \downarrow 0} \frac{\frac{k}{2} \epsilon^{-k / 2-1}}{-\frac{D}{2} \epsilon^{-1-1 / 2} \exp \left(D \epsilon^{-1 / 2}\right)} \\
& =\lim _{\epsilon \downarrow 0} \frac{\frac{k}{2} \epsilon^{-k+1 / 2}}{-\frac{D}{2} \exp \left(D \epsilon^{-1 / 2}\right)} .
\end{aligned}
$$

After applying the de l'Hospital rule $k-1$ more times, we get $\exp \left(-D \epsilon^{-1 / 2}\right)$ $=o(k / 2)$. Using Lebesgue's dominated convergence theorem once again we obtain the desired result.

\section{References}

[1] K. B. Athreya and S. N. Lahiri, Measure Theory and Probability Theory, Springer Science \& Business Media, 2006.

[2] M. W. Brandt and Q. Kang, On the relationship between the conditional mean and volatility of stock returns: A latent VAR approach, J. Financial Econom. 72 (2004), $217-257$.

[3] M. Brenner, R. Eldor, and S. Hauser, The price of options illiquidity, J. Finance 56 (2001), 789-805.

[4] U. Cetin, R. Jarrow, P. Protter, and M. Warachka, Pricing options in an extended Black Scholes economy with illiquidity: Theory and empirical evidence, Rev. Financial Stud. 19 (2006), 493-529.

[5] R. Cont, Empirical properties of asset returns: stylized facts and statistical issues, 2001.

[6] J. C. Cox and S. A. Ross, The valuation of options for alternative stochastic processes, J. Financial Econom. 3 (1976), 145-166.

[7] F. Delbaen and H. Shirakawa, A note on option pricing for the constant elasticity of variance model, Asia-Pacific Financial Markets 9 (2002), 85-99.

[8] G. Di Nunno, B. K. Øksendal, and F. Proske, Malliavin Calculus for Lévy Processes with Applications to Finance, Springer, 2009.

[9] L. C. Evans, Partial Differential Equations, Grad. Stud. Math. 19, Amer. Math. Soc., 1998.

[10] L. R. Glosten, R. Jagannathan, and D. E. Runkle, On the relation between the expected value and the volatility of the nominal excess return on stocks, J. Finance 48 (1993), 1779-1801.

[11] P. S. Hagan, D. Kumar, A. S. Lesniewski, and D. E. Woodward, Managing smile risk, Wilmott Magazine 2002, 84-108.

[12] D. Heath and M. Schweizer, Martingales versus PDEs in finance: an equivalence result with examples, J. Appl. Probab. 37 (2000), 947-957.

[13] Y.-L. Hsu, T. I. Lin, and C. F. Lee, Constant elasticity of variance (CEV) option pricing model: Integration and detailed derivation, Math. Computers Simul. 79 (2008), 60-71. 
[14] N. Ikeda and S. Watanabe, Stochastic Differential Equations and Diffusion Processes, Elsevier, 2014.

[15] H. Kunita, Stochastic differential equations and stochastic flows of diffeomorphisms, in: École d'Été de Probabilités de Saint-Flour XII-1982, Springer, 1984, 143-303.

[16] H. Liu and J. M. Yong, Option pricing with an illiquid underlying asset market, J. Economic Dynam. Control 29 (2005), 2125-2156.

[17] F. A. Longstaff, Option pricing and the martingale restriction, Rev. Financial Stud. 8 (1995), 1091-1124.

[18] P. A. Meyer, Probability and Potentials, Blaisdell, Waltham, MA, 1966.

[19] S. K. Nawalkha and N. A. Beliaeva, Efficient trees for CIR and CEV short rate models, J. Alternative Investments 10 (2007), 71-90.

[20] B. Øksendal, Stochastic Differential Equations, Springer, 2003.

[21] L. C. G. Rogers and S. Singh, The cost of illiquidity and its effects on hedging, Math. Finance 20 (2010), 597-615.

[22] M. Schroder, Computing the constant elasticity of variance option pricing formula, J. Finance 44 (1989), 211-219.

[23] Q. Wu, Series expansion of the SABR joint density, Math. Finance 22 (2012), 310345 .

Krzysztof Turek

Institute of Mathematics

Jagiellonian University

30-348 Kraków, Poland

E-mail: krzysztof.turek@uj.edu.pl 
